\title{
Comportement des matériaux cellulaires sous sollicitations dynamiques. Partie 2 : approche multi-échelles
}

\author{
PhiLIPpe Viot ${ }^{\mathrm{a}}$ \\ LAMEFIP, Arts et Métiers PARISTECH, Esplanade des Arts et Métiers, 33405 Talence Cedex, France
}

Reçu le 22 janvier 2010, accepté le 23 mai 2010

\begin{abstract}
Résumé - La méthodologie proposée pour l'étude du comportement de matériaux cellulaires sous sollicitation dynamique est abordée par une approche multi-échelles. Le comportement des mousses polymères étudiées dépend du matériau constitutif, et de sa structure poreuse. Les matériaux cellulaires de l'étude sont constitués de grains millimétriques poreux; le cœur de ces grains est constitué de cellules microscopiques. La méthodologie proposée vise à reproduire la morphologie multi-échelles du matériau cellulaire, celles des grains et des cellules, d'implémenter des modèles de comportement simples à ces différentes échelles afin de reproduire les phénomènes physiques complexes observés et la réponse macroscopique du matériau. L'observation et l'analyse des phénomènes locaux est un travail préparatoire à la modélisation multi-échelles. Cet article décrit les méthodes expérimentales et numériques qui ont été développées pour l'observation fine de la structure des matériaux cellulaires, et quantifier des déformations et des dommages de ces structures à l'échelle des cellules et des grains. Deux approches de modélisation ont été ensuite envisagées ; un modèle éléments-finis pour décrire la structure des grains, et une autre approche numérique plus originale qui s'appuie sur la méthode des éléments discrets pour représenter la structure microscopique des cellules.
\end{abstract}

Mots clés : Matériaux cellulaires / mousses / microtomographie / modélisation multi-échelles / modélisation éléments-finis / modélisation éléments discrets

\begin{abstract}
Behaviour of cellular material under dynamic loadings. Part 2: a multi-scale approach. A multi scale methodology is proposed for the study of the cellular material behavior under dynamic loading. The behavior of polymeric foams studied depends on the constitutive material and the morphology of the porous structure. The cellular material of this study is constituted of millimetric porous beads, these beads are themselves constituted of microscopic closed cells. The methodology proposed to model the multi scale morphology of the structure (the scales of beads and cells) of the cellular material; it consists in implementing simple mechanical models at the different scales to reproduce the complex physical phenomenon observed and to model the macroscopic response of the foam. The observation and the analysis of physical phenomenon is the first step of the multi scale modeling. This paper describes the experimental and numerical methods used to observe and describe the structure of the cellular material, and quantify deformations and damages of these structures at the scales of beads and cells. Two ways of modeling were investigated: a finite-element model to represent the bead structure and another approach, more original, by the use of a modified discrete element model to model the microscopic structure of the cells.
\end{abstract}

Key words: Cellular material / foams / microtomography / multi-scale modeling / finite-element model / discrete element model

\section{Introduction : du matériau à sa structure}

La modélisation des matériaux cellulaires est un des axes de recherche qui intéresse de plus en plus les

\footnotetext{
a Auteur pour correspondance :

philippe.viot@lamef . bordeaux.ensam.fr
}

industriels puisque ces matériaux sont utilisés dans de nombreux domaines (transport civil ou militaire, génie civil, équipement de sport, médical...) pour alléger des structures, atténuer un bruit parasite, isoler thermiquement des bâtiments ou dissiper une grande part de l'énergie d'un impact dans le cadre de la sécurité passive. 
La volonté de développer des modèles numériques dédiés à ces matériaux n'est pas nouvelle puisqu'elle répond à des préoccupations techniques et économiques. Plusieurs voies de modélisation peuvent être envisagées; une voie classique de modélisation du comportement du matériau à l'échelle macroscopique et une seconde voie de modélisation - dite multi-échelles prenant en compte les phénomènes rencontrés aux échelles plus fines de la structure du matériau.

L'approche macroscopique qui vise à décrire la réponse contrainte déformation du matériau homogénéisé, en prenant en compte les paramètres tels que la densité de la mousse, la vitesse de déformation imposée et les modes de sollicitation, a déjà été décrite dans un article précédent [1]. Cependant, les modèles macroscopiques envisagés ne permettent pas par nature de décrire les phénomènes locaux observés lors de compression dynamique sur des matériaux cellulaires (flambement des parois dans le cas de mousses polymères, perforations et ruptures de parois, localisation des déformations) et une approche micro-macro doit être envisagée. Ce deuxième article présente une deuxième voie d'investigation qui vise à proposer des modèles reliant la morphologie de la structure de ces matériaux cellulaires à leur comportement macroscopique. Par cette approche, il sera donc possible d'élaborer une mousse, c'est-à-dire de choisir un matériau constitutif et définir un procédé d'élaboration, pour obtenir une structure particulière afin d'atteindre des performances macroscopiques attendues. Cet objectif intéresse aussi les industriels pour une meilleure maîtrise des matériaux utilisés dans leurs produits.

La deuxième voie d'investigation propose donc de reproduire la morphologie multi-échelles du matériau cellulaire, celles des grains et des cellules, d'implémenter des modèles de comportement simples à ces différentes échelles afin de reproduire les phénomènes physiques observés et la réponse macroscopique du matériau. L'observation et l'analyse des phénomènes locaux est un travail préparatoire à la modélisation multi-échelles. Cet article décrit les démarches qui ont été menées pour l'observation fine de la structure des matériaux cellulaires, des déformations et des dommages de ces structures à l'échelle des cellules (échelle microscopique) et des grains (échelle mésoscopique). L'objectif est de qualifier les paramètres influant sur ces phénomènes locaux de dégradation du matériau afin de les prendre en compte dans les modélisations multi-échelles. Le résultat majeur de cette analyse des phénomènes aux différentes échelles est de considérer un essai de caractérisation, non pas comme un essai rhéologique, mais comme un essai sur structure. Pour certains matériaux, les localisations de déformation sont telles que le corps d'épreuve sollicité ne peut plus être considéré comme un échantillon mais comme une structure.

De ces observations et scénarii de propagation de dommages proposés aux deux échelles mésoscopique et microscopique, deux approches de modélisation ont été envisagées; un modèle éléments-finis qui consiste à décrire la structure mésoscopique de la mousse comme un assemblage de parois de grains (dans ce cas, la porosité du grain a été modélisée par un matériau poreux homogénéisé), et une autre approche numérique qui s'appuie sur la méthode des éléments discrets pour représenter la structure des cellules (échelle microscopique) et évaluer l'influence de la variabilité de la morphologie de la structure cellulaire à cette échelle.

\section{Analyse des dommages aux différentes échelles}

Les observations à l'aide de caméra rapide et les mesures par corrélation d'images faites à la surface de l'échantillon [1] lors de compressions statique et dynamique montrent des localisations en déformation et en température qui traduisent des mécanismes localisés de dommage localisés. Ces premières investigations doivent être poursuivies au cour du matériau en utilisant d'autres techniques expérimentales pour décrire la progression des mécanismes d'endommagement aux différentes échelles de la structure du matériau.

Les structures des matériaux cellulaires ont déjà fait l'objet de nombreuses publications tant leurs effets sur le comportement macroscopique d'un point de vue thermique, phonique ou mécanique est important. Dans un grand nombre de publications (de la chimie des matériaux ([2-4]), aux procédés [5] et à la mécanique) dédiées à ces matériaux, on retrouve au moins une image MEB de la structure du matériau pour illustrer l'importance du procédé d'élaboration sur la structure générée ou l'effet de cette structure sur la réponse macroscopique. De nombreux articles décrivent ces microstructures et les ouvrages de référence de Gibson et Ashby [6] et de Mills [7] font une bonne synthèse des microstructures que l'on peut retrouver dans les matériaux cellulaires existants. La méthodologie proposée - de la structure du matériau à son comportement macroscopique - nécessite l'identification fine de la microstructure des matériaux polymères de l'étude et le recours à l'expertise d'images MEB ou reconstruites en micro tomographie est aussi indispensable.

\section{1 À l'échelle microscopique}

Les matériaux cellulaires de cette étude sont principalement des mousses polymères (Figs. 1a , b et e, obtenues par moussage lors d'une réaction chimique, fabricants JSP S.A. et Knaupf), des mousses syntactiques (réalisées par l'assemblage de billes creuses dans une matrice époxyde, Fig. 1d), des mousses biologiques ou naturelles (liège et bois, Fig. 1f) et, à titre plus anecdotique, des mousses métalliques (Fig. 1c). L'ensemble de ces mousses est constitué d'une première échelle (dite microscopique) de cellules ouvertes (liège, bois - Fig. 1f, polyuréthane Fig. 1b, polystyrène...) ou fermées (polypropylène Fig. 1e, polystyrène, aluminium - Fig. 1c...) de tailles et d'épaisseurs de parois variables (Fig. 1). Ces dimensions dépendent des propriétés du matériau constitutif 

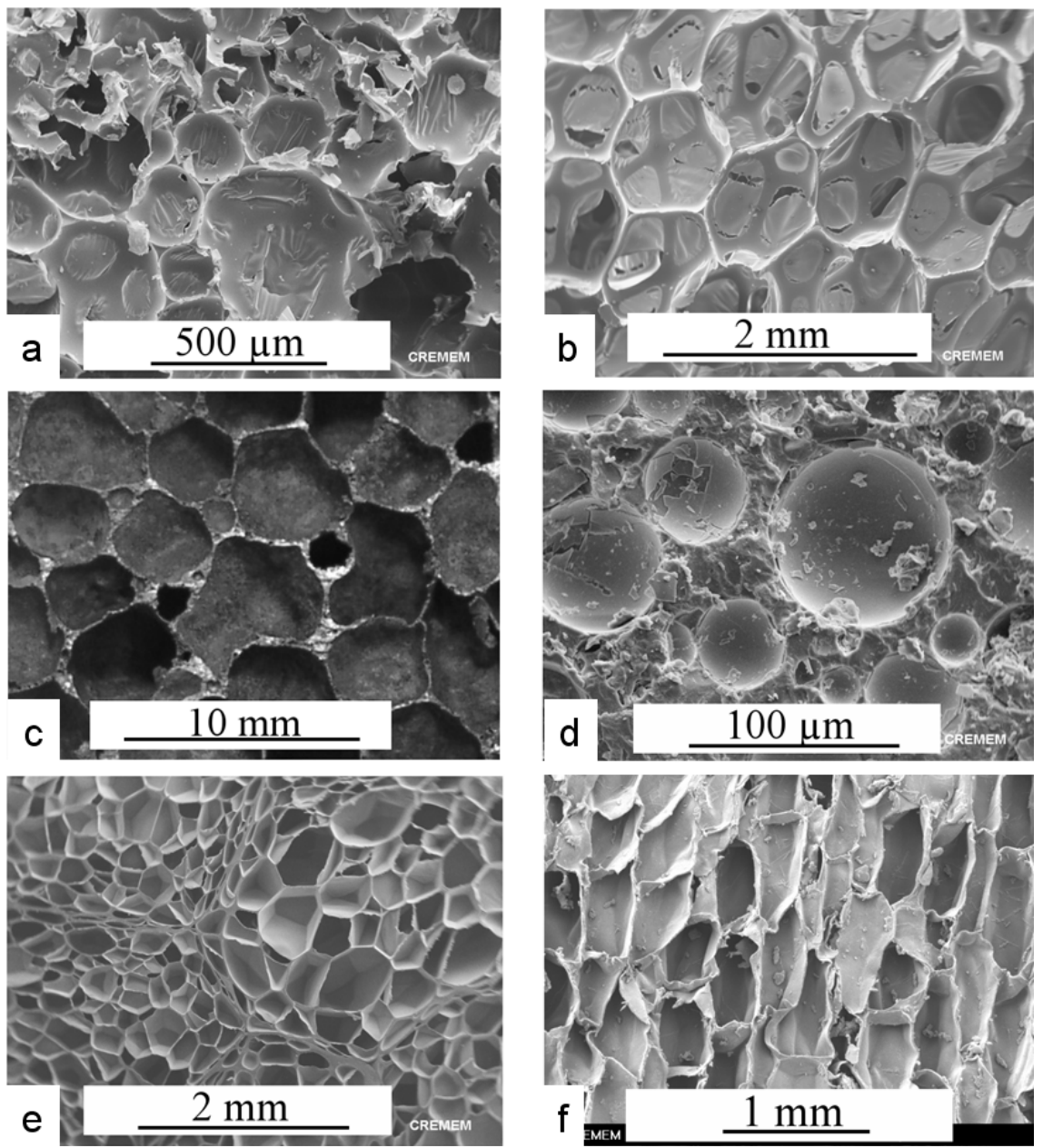

Fig. 1. Vue micrographique de structures cellulaires de mousses de polyuréthane (a et b), d'aluminium (c), syntactique (d), de polypropylène (e) et de liège (f).

et des procédés de fabrication. Pour le même matériau constitutif, du polystyrène par exemple, les procédés en émulsion [8] permettent d'obtenir des mousses calibrées à porosités ouvertes dont les tailles de cellules sont inférieures à la dizaine de microns. À partir du même matériau, une mousse à porosité fermée, dont la taille des cellules est cette fois d'une dizaine à une centaine de microns, peut être réalisée par moussage obtenu par voie $\mathrm{CO}_{2}$ super critique [9]. Pour les mousses syntactiques, la taille des cellules dépend intrinsèquement de la taille des billes creuses incluses dans la matrice (Fig. 1d); elle peut être de quelques dizaines de microns dans le cas de microsphères creuses de verre et peut atteindre quelques dizaines de millimètres dans le cas d'assemblages de billes creuses polymères (fabricant ATECA S.A.).

À l'échelle macroscopique, le comportement classique (en terme de contrainte-déformation) d'un matériau cellulaire sous une sollicitation de compression est caractérisé par trois phases (Fig. 2a) ; une phase élastique limitée par une contrainte seuil $\sigma_{\mathrm{s}}$ suivie par un plateau en contrainte (appelé abusivement plateau plastique) et une phase ultime de densification pendant laquelle la contrainte augmente fortement en fonction de la déformation. Ce comportement de matériaux cellulaires sous compression est classique, il a été présenté par de nombreux auteurs [1] pour des mousses polymères (Fig. 2a) et dans de nombreux ouvrages de référence $[6,7]$. Ce comportement macroscopique est aussi révélé pour des matériaux cellulaires plus originaux tels que les mousses syntactiques ou des assemblages de billes creuses (Fig. 2b). Il est bien évident que la taille des cellules, leurs géométries, leurs assemblages et le matériau constitutif conditionnent le comportement de la mousse. Dans un premier temps, afin de considérer le comportement de la structure à l'échelle microscopique, des mousses mono échelle (PU, mousse d'aluminium ou mousse syntactique) ont fait l'objet d'une expertise et révèlent des comportements très différents :

- La compression d'une mousse d'aluminium génère des ruptures dans les parois des cellules. Ces parois viennent ensuite indenter d'autres parois non endommagées pour initier de nouvelles ruptures. Cette réponse bien différente d'une mousse polymère est due en grande partie au comportement de l'aluminium.

- Dans le cas des mousses syntactiques, le comportement macroscopique et microscopique dépend fortement de la fraction volumique de sphères creuses 


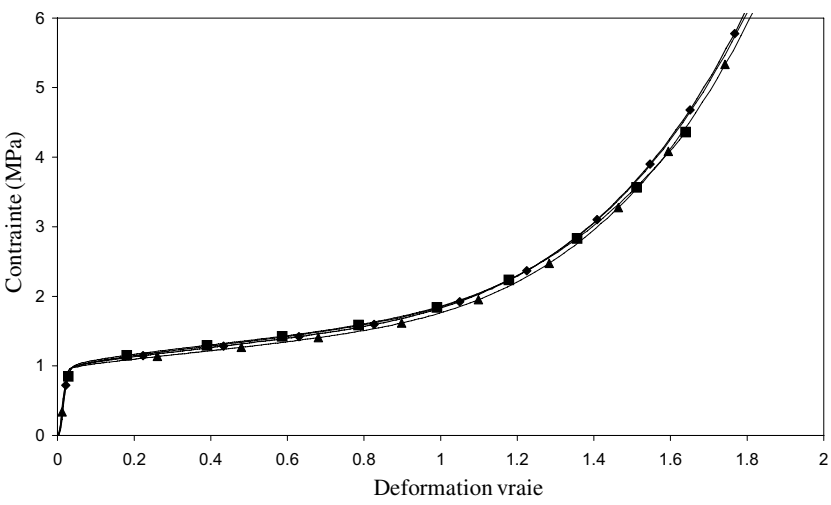

(a)

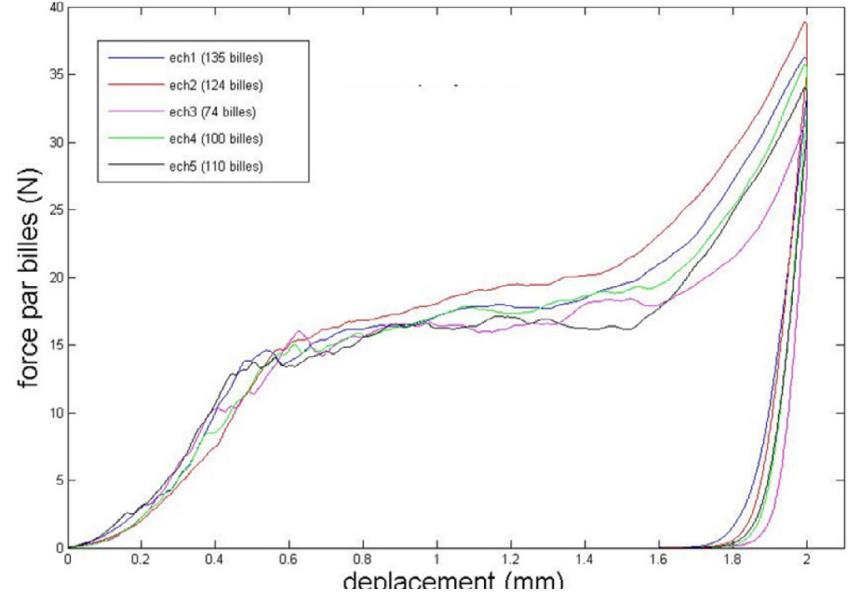

(b)

Fig. 2. (a) Comportement d'une mousse de polypropylène (densité $80 \mathrm{~kg} \cdot \mathrm{m}^{-3}$ ) sous compression statique. (b) Réponse macroscopique d'un matériau cellulaire constitué de billes creuses sous compression statique.

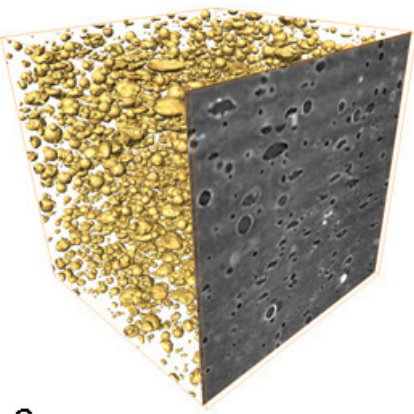

a

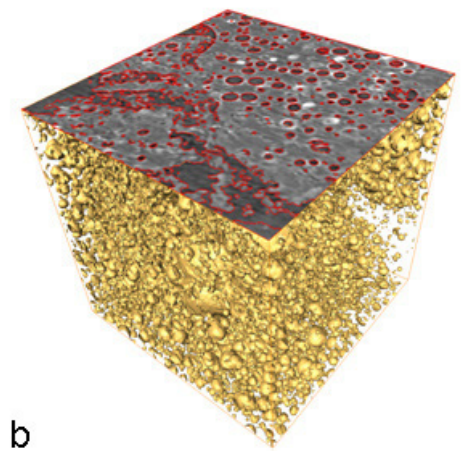

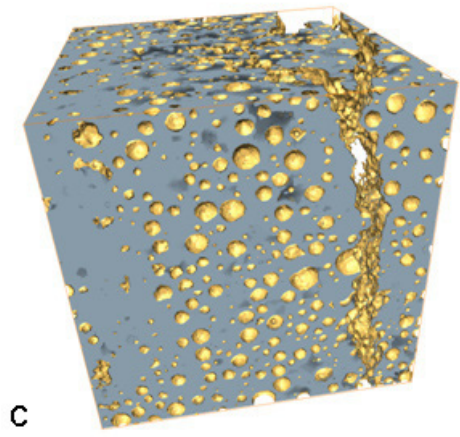

Fig. 3. Reconstruction micro tomographique après compression dynamique d'une mousse syntactique de trois fractions volumiques de sphères $15 \%$ (a), $30 \%$ (b), $45 \%$ (c).

dans la matrice; il dépend donc fortement de sa structure. Pour des faibles fractions de billes, on assiste à une déformation viscoélastique de la matrice et aux écrasements localisés de sphères (Fig. 3a). Pour de fortes proportions de sphères creuses, le matériau devient fragile (Fig. 3c). Sa dégradation apparaît par la propagation de fissures qui progressent entre les « défauts » que constituent les inclusions de sphères. Pour des fractions intermédiaires (Fig. 3b), les mécanismes de dégradation du matériau sont mixtes, des bandes d'endommagement (où les sphères creuses sont écrasées) et des fissures apparaissent. Pour ce type de matériau, il y a donc un couplage entre l'effet de structure (organisation des billes) et l'effet matériaux constitutifs (la proportion de mélange matrice/sphères est couplée à la microstructure).

- La compression d'une mousse de polyuréthane induit le flambement des parois des cellules et l'apparition de rotules plastiques. Ces déformations s'accompagnent aussi de déchirements de parois initiés par la combinaison des sollicitations mécaniques et des différentiels de pression du gaz comprimé dans les cellules. Les déformations sont plastiques puisque les dommages peuvent être observés après sollicitation, ce qui n'est pas le cas des mousses de néopolène structurellement équivalentes mais dont le matériau constitutif est viscoélastique (le retour à la structure initiale se manifeste de manière différée).

Dans le cas des mousses polymères multi-échelles, qui font plus particulièrement l'objet de cet article, les mécanismes de déformations tels que le flambement et la rotule plastique sont aussi présents. Les observations par MEB montrent clairement les flambements générés sur les parois des cellules (Fig. 4). Les déformations résiduelles observées sont variables; certaines cellules semblent n'avoir été que faiblement déformées (cellule A, Fig. 4) alors que d'autres ont atteint un niveau de déformation correspondant à la phase de densification (cellule B, Fig. 4).

De ces observations, une hypothèse de scénario peut être envisagée en termes de propagation du dommage. On peut tout d'abord supposer qu'il n'existe pour la cellule que les deux phases d'élasticité et de densification; la progressivité du passage de la phase élastique 


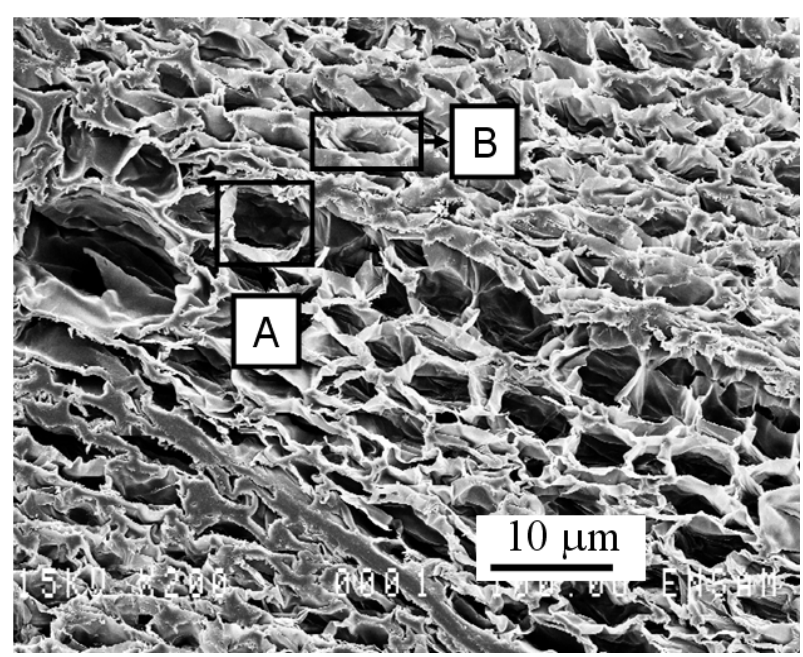

Fig. 4. Photographie MEB d'une mousse PP après compression dynamique : apparition de bande de localisation de dommage à l'échelle de la cellule.

à la densification dépend évidemment de la fragilité du matériau constitutif, certainement aussi de la géométrie des cellules, de la contribution du gaz sous pression contenu dans le volume de la porosité et de la vitesse de la sollicitation. Pendant la sollicitation de compression exercée sur la mousse, au début de la phase plastique (macroscopiquement parlant), un ensemble de cellules s'endommage, leurs parois flambent et les porosités se ferment partiellement (voire totalement en fonction de la pression du gaz contenu dans les cellules). La densification locale partielle ou non - d'un ensemble de porosités permet la dissipation de l'énergie de la sollicitation, le reste du matériau cellulaire conservant son élasticité. Le plateau plastique observé macroscopiquement s'explique alors facilement par la fermeture des porosités qui se propage sur l'ensemble de l'échantillon pendant ce plateau. La densification ultime (macroscopique) correspond finalement à la dégradation de l'ensemble des cellules.

De plus, ces observations montrent que la dégradation du matériau s'effectue par strates (souvent perpendiculaires à l'axe de la sollicitation, Figs. 4 et 5). Le phénomène de localisation apparaît principalement pour les mousses multi-échelles où l'influence des parois des grains peut être suspectée sur l'état de contrainte local imposé à la structure cellulaire. La propagation du dommage peut être expliquée en considérant une cellule de caractéristiques mécaniques plus faible «effet de taille » ou plus sollicitée «effet de structure multi-échelles ». Sous la sollicitation, les parois de cette cellule flambent ce qui induit une redistribution et une augmentation de la charge sur les cellules voisines. Par effet « boule de neige », on assiste alors à un effondrement qui se propage dans la structure cellulaire voisine.

Pendant ces processus, il est difficile de mettre en évidence sur le comportement des matériaux cellulaires industriels l'effet de la taille et de la géométrie des cellules (la dispersion des tailles de cellules est trop importante pour les mousses polymères concernées). Des

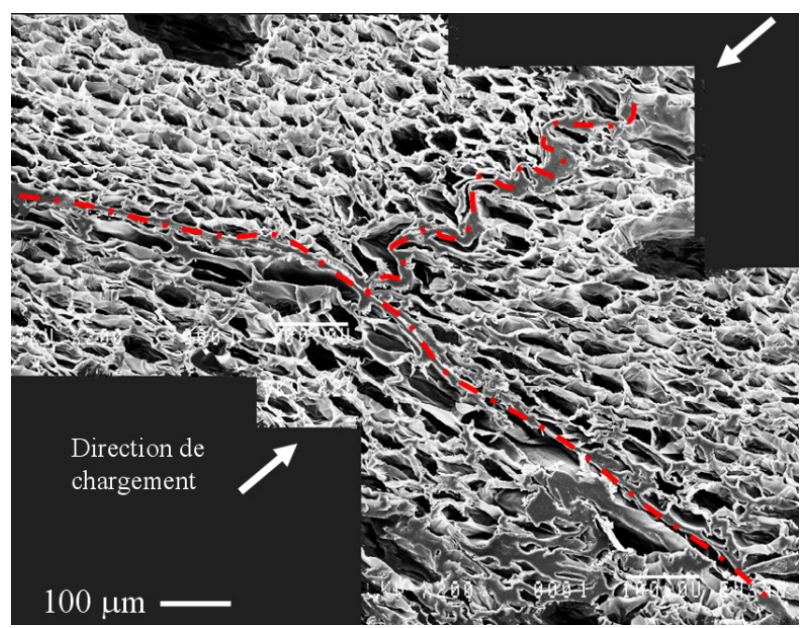

Fig. 5. Photographie MEB d'une mousse PP après compression dynamique : apparition de bandes de localisation de dommage et de flambement multi modes à l'échelle du grain.

études expérimentales complémentaires sont en cours sur des mousses de densité constante et de taille de porosité contrôlée afin de mettre en évidence l'effet de taille des cellules sur la localisation du dommage. Les modélisations multi-échelles amèneront aussi un certain nombre de réponses concernant le processus de propagation du dommage dans ce type de matériau.

\section{2 À l'échelle mésoscopique}

Les matériaux cellulaires polymères de l'étude sont constitués de grains agglomérés. Le réseau des parois de ces grains forme une structure à une échelle supérieure désignée arbitrairement par mésoscopique (Fig. 6). Pour des raisons de simplification, les parois de cette structure sont considérées denses et homogènes. Cependant, lors de l'élaboration du matériau cellulaire, ce sont les cellules en périphérie de chaque grain qui sont déformées, écrasées et fondues pour constituer une paroi supposée dense (Fig. 7). Une analyse micrographique plus fine montrerait certainement encore la texture de ces parois engendrée par l'écrasement des cellules. Il est d'ailleurs possible d'observer des porosités dans les parois (aux endroits où les pressions de contact ont été les moins fortes pendant l'élaboration). Dans certains cas, pour des mousses de faibles densités, il est même difficile d'isoler la paroi constitutive de la structure mésoscopique de l'échelle microscopique des cellules, tant le différentiel en épaisseur est faible entre les parois de grain et de cellule; seul un gradient de taille de cellules peut être observé à la frontière des grains (Fig. 1e).

\subsubsection{Analyse 2D des localisations de déformation}

Les observations des dégradations de la structure par un chargement dynamique ont été effectuées en microscopie électronique à balayage et par micro tomographie. Les 


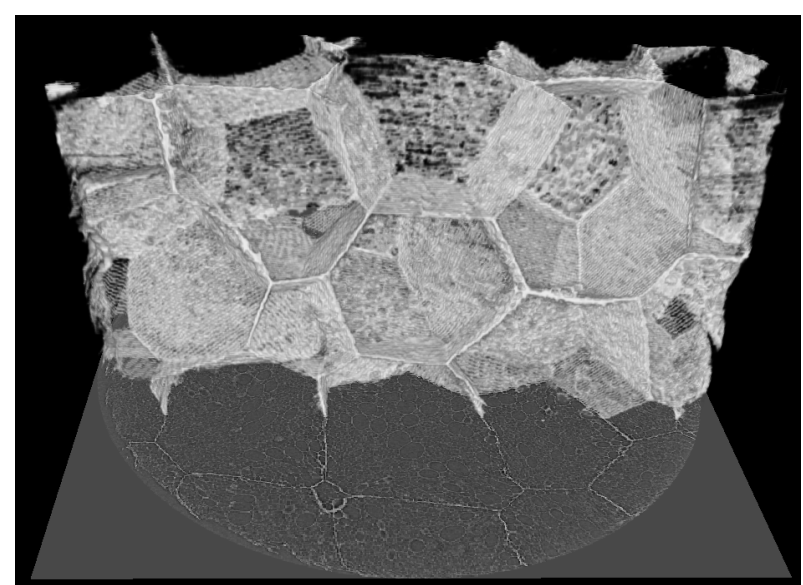

Fig. 6. Reconstruction micro tomographique d'un ensemble de parois de grains de mousse PP.

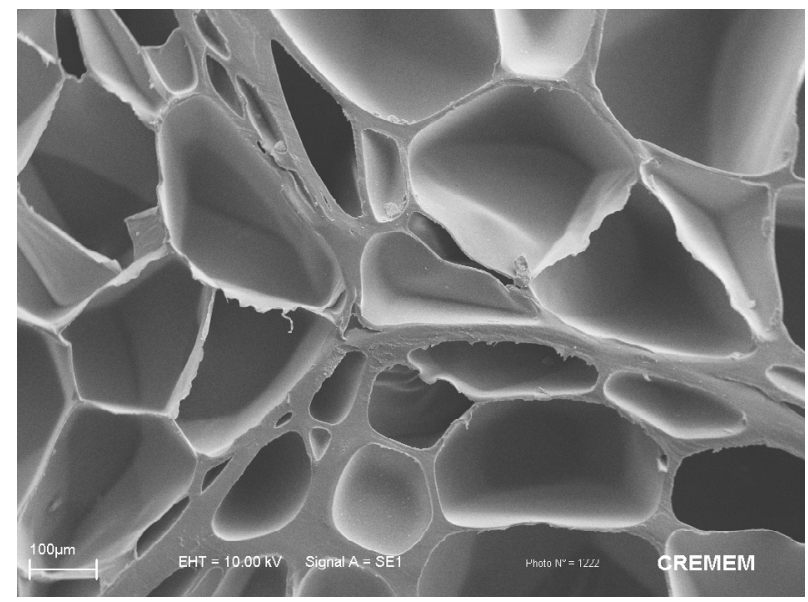

Fig. 7. Image MEB d'une mousse PP.

images obtenues par MEB sur des échantillons découpés après impact révèlent les déformations résiduelles de la structure cellulaire. La déformation des parois des grains dépend de leur orientation par rapport à l'axe de sollicitation. En compression uni axiale, les modes de déformation sont la flexion et le flambement (qui intervient en toute logique sur les parois dont les directions sont proches de l'axe de compression). Et puisque ces parois sont maintenues latéralement par les cellules (échelle microscopique), le flambement est multi modal. Les nœuds de flambement doivent apparaître en fonction de la variabilité de l'épaisseur de paroi mais dépendent aussi de la rigidité latérale locale de la structure cellulaire. La densité locale de la structure microscopique (et donc sa rigidité face à des sollicitations dynamiques) influent donc sur la déformation de la structure mésoscopique; il y a bien évidemment interdépendance des déformations observées aux deux échelles.

Ces observations au MEB ont été faites après découpe de l'échantillon et, comme dans bien des cas, l'observation (ou la mesure) peut perturber le phénomène observé. La découpe d'une mousse polymère n'est pas simple et peut générer des dommages sur la structure cellulaire équivalents à ceux engendrés par la sollicitation dynamique. Des moyens d'instrumentation complémentaires devaient être développés pour observer ces phénomènes au cœur de la structure sans découpe préalable. L'utilisation de la technique de micro tomographie a donc été proposée. Cette technique de mesure permet de caractériser en trois dimensions la structure d'un matériau hétérogène avec une grande précision mais est incompatible avec les moyens expérimentaux usuels d'impact. Les instruments de mesure en micro tomographie (table micrométrique multiaxiale, caméra haute définition...) sont fragiles et coûteux et ne peuvent être utilisés directement sur une machine de sollicitation dynamique conventionnelle. De plus, les temps de mesure sont longs (souvent supérieurs à $60 \mathrm{~min}$ ), la mesure ne peut être donc réalisée pendant le temps de l'impact (inférieur à quelques millisecondes).

L'identification en 3D du champ de déformation de la mousse et de la propagation du dommage par flambement des parois des cellules était nécessaire dans la démarche de modélisation multi-échelles. Des moyens expérimentaux de sollicitation dynamique compatibles avec les contraintes techniques des mesures par micro tomographie ont donc été développés [10]. La méthode expérimentale retenue consiste à réaliser des essais d'impact interrompus suivis de mesure de micro tomographie. Une première acquisition micro tomographique est effectuée sur la structure saine de la mousse, avant impact. L'échantillon est ensuite impacté en utilisant une tour de chute. Pendant le chargement dynamique, l'amplitude de déformation est limitée à une certaine valeur. L'échantillon est maintenu comprimé et est ensuite placé une seconde fois sur la table de mesure de micro tomographie pour être radiographié. Ces opérations (impacts et acquisitions micro tomographiques) sont répétées jusqu'à la complète densification de la mousse. La déformation du matériau cellulaire peut être évaluée à partir des reconstructions 3D des images de micro tomographie enregistrées aux différentes étapes de l'essai de compression dynamique. Il était nécessaire de vérifier si cette méthodologie (impact interrompu suivi d'un long temps de mesure pendant lequel le matériau est maintenu comprimé) ne modifiait pas le comportement de la mousse. Des cycles de compression ont été effectués pour mettre en évidence que la méthodologie d'essais interrompus ne modifiait pas la réponse macroscopique du matériau cellulaire.

Enfin, pour cette étude, parce que les impacts devaient être appliqués sur des volumes d'échantillon représentatifs de la structure de la mousse (donc les plus grands possible) mais aussi parce que la précision de mesure micro tomographique est d'autant meilleure que l'échantillon est petit, le diamètre de l'échantillon a été fixé à $10 \mathrm{~mm}$. Ce choix a permis de mesurer avec une bonne précision la géométrie des parois des grains (échelle mésoscopique). En revanche, la précision de mesure ne permet pas d'identifier la structure des cellules (échelle microscopique).

La technique de micro tomographie a été appliquée à une mousse de polypropylène. Les mesures ont été effectuées sur la ligne BM05 du synchrotron européen ESRF 


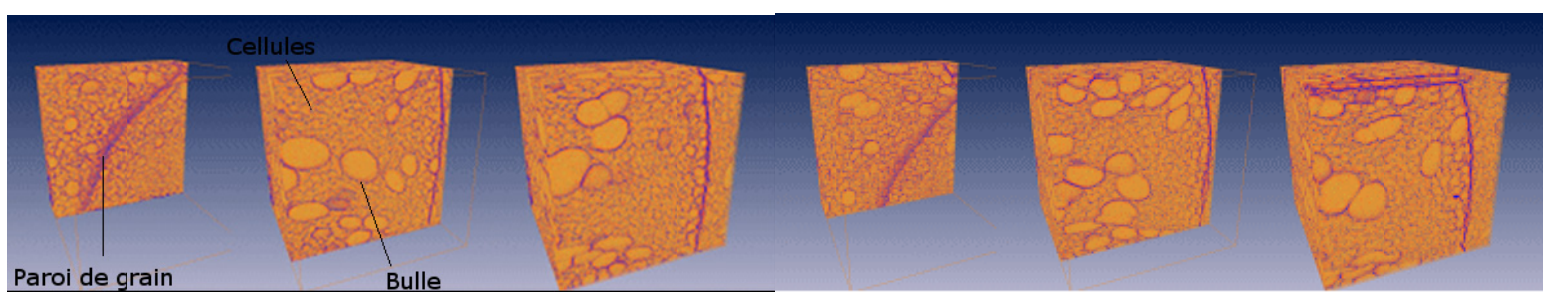

(a)

(b)

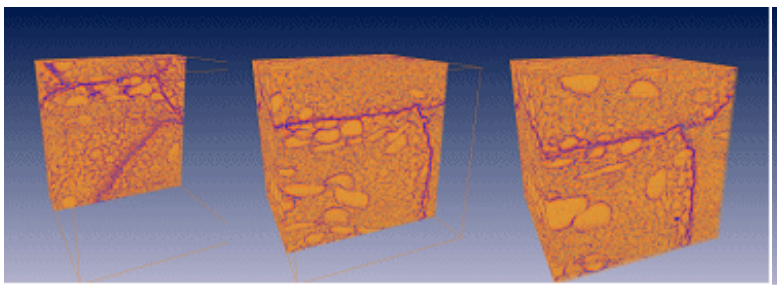

(c)

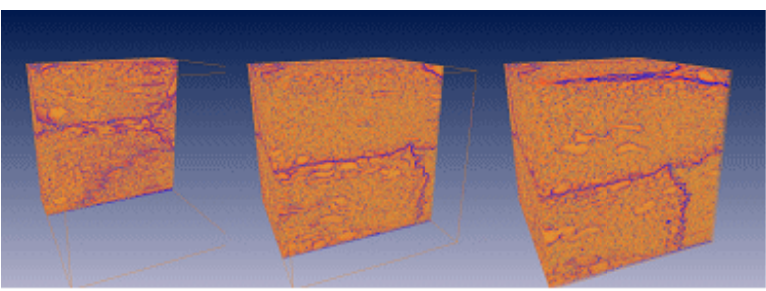

(d)

Fig. 8. Reconstruction micro tomographique d'un cube (de coté $2 \mathrm{~mm}$ ) situé dans la partie basse de l'échantillon impacté. (a) Avant impact, (b) après une déformation macroscopique imposée de $10 \%$, (c) après une déformation macroscopique imposée de $30 \%$, (d) après une déformation macroscopique imposée de $50 \%$.

à Grenoble. Les reconstructions 3D de l'échantillon de mousse permettent de visualiser correctement les parois des grains ainsi que les larges inclusions de bulles d'air (Fig. 8). À une échelle beaucoup plus fine, il est possible de distinguer aussi la structure des cellules fermées, il est cependant impossible de quantifier ces observations, puisque la précision des mesures est de l'ordre de grandeur de l'épaisseur des parois des cellules (la taille des figures présentées dans l'article ne permet pas de visualiser cette échelle).

La reconstruction 3D d'un cube situé dans la partie inférieure de l'échantillon montre les différents mécanismes de déformation (Fig. 8). Les premiers flambements apparaissent dans cette zone à partir d'une déformation imposée de $30 \%$ (Fig. 8c) ; ce sont les parois verticales dans l'axe de la compression, qui sont déformées suivant ce mode; les autres parois subissent principalement de la flexion. S'il est impossible de distinguer la déformation des cellules sur ces premières figures, l'écrasement des bulles plus larges peut être visualisé. Pour une déformation de $50 \%$ (Fig. 8d), ces bulles sont complètement écrasées ce qui tend à prouver que le matériau cellulaire a localement atteint la phase de densification.

Ces résultats sont confirmés sur les coupes verticales de l'échantillon (Fig. 9). Une première analyse des déformations peut être faite à partir de ces images $2 \mathrm{D}$ si on suppose que le déplacement hors plan est nul (cette hypothèse a été validée puisque le déplacement des grains est principalement vertical, le déplacement radial peut être considéré comme négligeable puisque le coefficient de Poisson de cette mousse est supposé proche de zéro). Un traitement numérique a été appliqué sur une partie de la figure 9 afin de mettre en relief les phénomènes observés. Ce même filtre a été appliqué sur les différentes sections

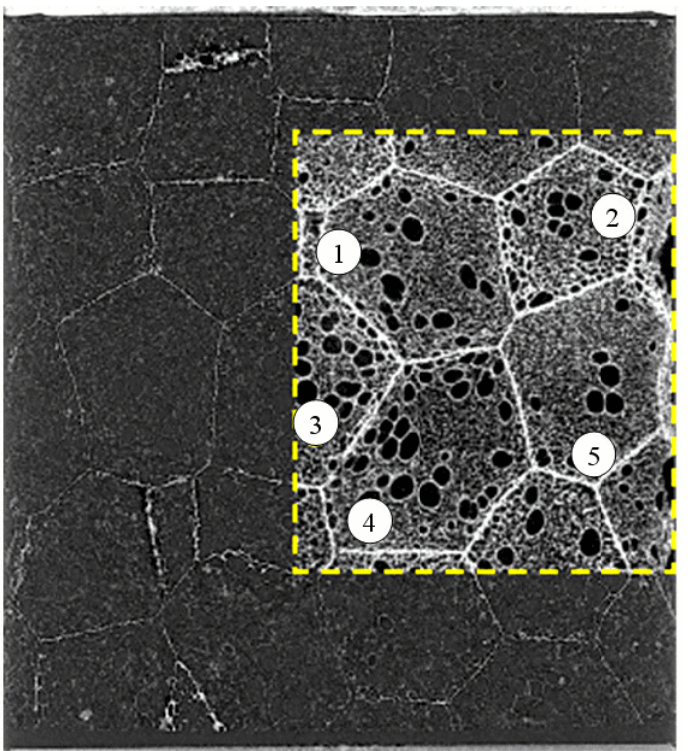

Fig. 9. Coupe verticale (obtenue en microtomographie d'un échantillon de mousse de polypropylène).

obtenues après chaque impact. Le résultat est présenté sur la figure 10 correspondant aux trois premiers impacts.

En première approximation, la variation de surface de chaque grain peut être considérée comme un bon indicateur de sa déformation moyenne. Cette estimation a été effectuée sur l'ensemble des grains d'une section et montre la forte dispersion des déformations sur les grains appartenant à cette coupe verticale (Fig. 9). La densité de chaque grain peut être aussi estimée à partir du niveau de gris de sa structure poreuse. Sachant que sur ces images, la teinte la plus claire correspond au matériau dense et qu'un pixel noir représente un point géométrique à l'intérieur d'une 

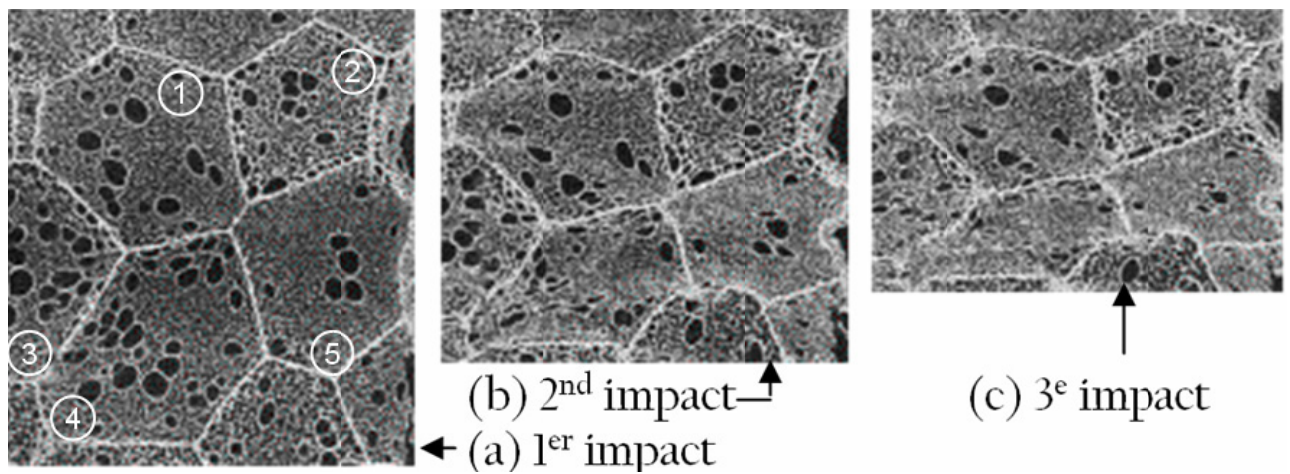

(c) $3^{\mathrm{e}}$ impact

Fig. 10. Coupe verticale de la zone délimitée de la figure 9 montrant les déformations consécutives à trois impacts.

porosité, le niveau moyen de gris d'un grain est corrélé à sa densité (cependant, la précision de mesure ne permet pas de déterminer exactement la densité des grains). Sur l'ensemble des grains présents dans la coupe verticale de l'échantillon, l'étude de ces deux indicateurs - variation de surface et niveau de gris moyen - montre qu'il n'y pas de corrélation entre la densité du grain et sa déformation. La structure mésoscopique constituée par le réseau de parois de grain a manifestement une influence significative sur la localisation de la déformation dans l'échantillon.

Ainsi, cette analyse de variation de surface de grain révèle une hétérogénéité dans la déformation moyenne de chaque grain. Ces images 2D montrent aussi des bandes de localisation de déformation à l'intérieur de chaque grain; ces bandes peuvent être identifiées (par exemple sur le grain 4 (Figs. 9 et 10) assez facilement en suivant la déformation des bulles d'air et la variation du niveau de gris dans le grain (les zones qui s'éclaircissent se densifient). Cette localisation de déformation s'accompagne de flambement de parois et d'indentation locale (des parois verticales dans l'axe de sollicitation s'enfoncent dans les parois perpendiculaires de grain inférieur). Enfin, le suivi de contour de grains montre que la déformation locale de la structure est complexe et est initiée par des champs de force autres que ceux générés par une simple compression macroscopique uni axiale.

\subsubsection{Analyse 3D des localisations de déformation}

Malgré l'ampleur des développements numériques et les coûts en temps de calcul, l'étude en 3D des images reconstruites en micro tomographie a été effectuée pour deux raisons principales : premièrement, il était nécessaire de vérifier sur l'ensemble de l'échantillon les conclusions déduites des observations faites sur quelques sections verticales qui nécessitaient de surcroît des hypothèses fortes. Deuxièmement, la reconstruction en 3D de la géométrie des grains est une étape essentielle pour la définition d'un modèle numérique d'une structure réelle de mousse, modèle qui pourra être ensuite implémenté dans un code de calcul.

Pour les raisons évoquées précédemment (porosité dans les parois des grains, épaisseur de paroi de grain équivalente à celle des bulles. . .), il a été particulièrement délicat d'extraire, par des techniques d'imagerie traditionnelles, le réseau constitué par les frontières des grains à partir des images 3D obtenues en micro tomographie. Les reconstructions des parois ainsi obtenues présentaient des épaisseurs très variables, des porosités et des trous, et il semblait donc impossible d'identifier à partir de cette structure 3D des domaines fermés représentant l'intérieur poreux des grains et le réseau de parois.

La géométrie de la structure des parois des grains ne pouvait être obtenue par une méthode directe, il a donc été proposé de développer une méthode de reconstruction afin de déterminer un Volume Représentatif de chaque Grain (désigné par VRG par la suite) sur lequel la déformation moyenne et la densité ont été estimées. La méthode a nécessité des développements d'algorithmes complexes de traitement d'images qui ne seront pas présentés dans ce document mais qui pourront être retrouvés dans la référence [11]. Pour expliquer succinctement la technique de détermination du VRG, une première série de filtres numériques est appliquée sur l'image 3D obtenue par micro tomographie pour en extraire approximativement les centres des grains. Pour chaque centre de grain, un algorithme génère une surface développable fermée qui est expansée à l'intérieur du grain et vient progressivement s'appuyer sur une frontière numérique délimitée par les parois des grains (la paroi est identifiée par son niveau de gris). L'ensemble des VRG poreux des grains a été calculé à chaque étape de la compression dynamique (Fig. 11) ; chaque VRG étant délimité par une surface développable continue, il a été possible d'estimer des grandeurs géométriques (barycentre, volume) et le niveau de gris moyen des pixels de ce VRG. La mesure de niveau de gris moyen dans ce VRG permet de déduire un densité moyenne $\rho$ sur le grain ou, par dualité, sa porosité $\phi$. Quelques équations permettent de retrouver une relation simple liant la déformation volumique du domaine VRG avec la variation de porosité entre son état non comprimé (indicé 0 ) et après chaque étape $(j)$ de la compression dynamique :

$$
\varepsilon_{j}^{\mathrm{vol}}=1-\frac{\phi_{j}-\phi_{0}}{1-\phi_{j}}
$$

Les résultats de ces calculs appliqués à l'ensemble des grains de l'échantillon de mousse (Fig. 11) et aux 


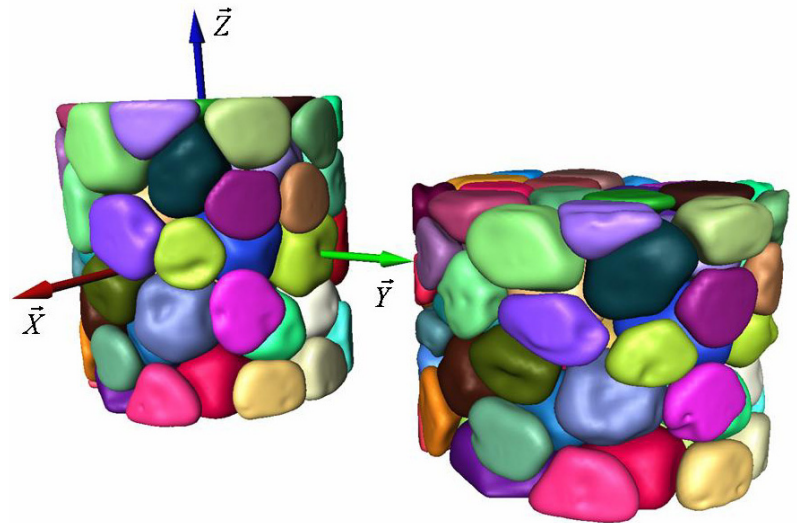

Fig. 11. Reconstruction de la morphologie des grains avant compression dynamique (à gauche) et après le second impact (à droite).

différents niveaux de la compression dynamique apportent des informations qui permettent de nuancer les conclusions faites lors des analyses 2D. Il existe effectivement une forte dispersion dans la déformation volumique des grains au début de la compression dynamique et ceci quelle que soit la densité (marqueurs carrés, Fig. 12). Malgré une légère tendance qui semble montrer que les grains les plus denses sont les moins déformés, on peut estimer que la densité n'est pas le facteur prépondérant. Une étude plus fine montre que pour ce niveau de déformation moyenne imposée, il existe une corrélation forte entre la déformation volumique des grains et leur position dans l'échantillon ce qui révèle à nouveau les phénomènes de localisation. La corrélation entre la déformation volumique et la densité du grain devient plus évidente pour des déformations moyennes supérieures; pour le deuxième et le troisième impact (marqueurs disque et losange, Fig. 12), une relation peut être présentée entre la densité du grain et sa déformation volumique : les grains plus denses sont plus rigides et leur déformation volumique est plus faible. De plus, la dispersion en déformation volumique est indépendante de la densité, et peut être considéré comme un effet de la structure des parois des grains qui initie des localisations de déformation lors de l'apparition de flambement. En fin de compression (dernier impact, marqueurs triangle, Fig. 12), la déformation moyenne imposée sur l'échantillon implique sa densification, la dispersion des déformations est alors beaucoup plus faible.

En conclusion, les observations faites aux différentes échelles montrent l'apparition de localisation de déformation pendant la compression; elles apparaissent à la surface du matériau, sont visibles après impact sur des photographies de la structure aux différentes échelles (obtenues par MEB ou par micro tomographie). Cette dégradation localisée s'accompagne (ou s'initie) par le flambement des parois des cellules (à l'échelle mésoscopique) et celles des grains (à l'échelle microscopique). Le même mécanisme est donc à l'origine de la dégradation des deux échelles du matériau.

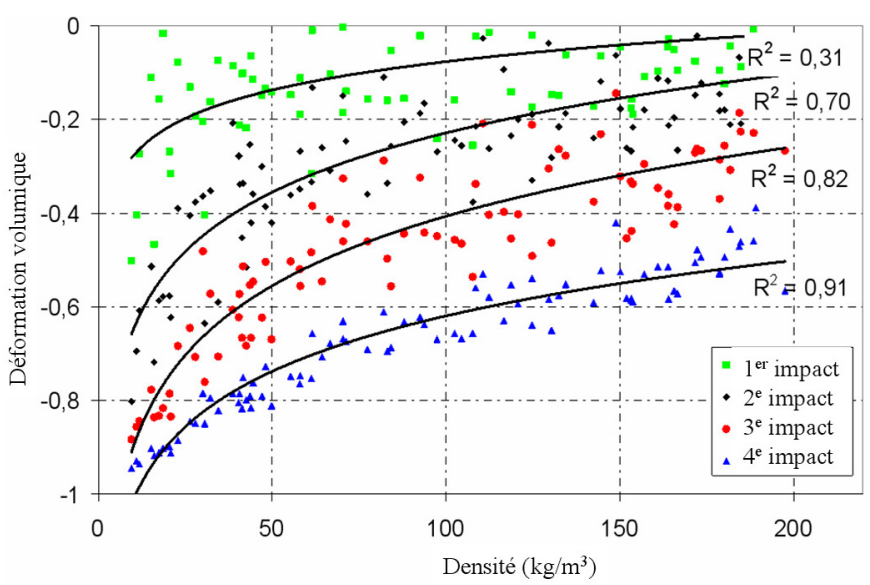

Fig. 12. Évolution de la déformation volumique des grains en fonction de leurs densités et à différentes étapes de la compression dynamique.

Les traitements numériques spécifiques des images obtenues par microtomographie ont fourni une morphologie des volumes représentatifs des grains. L'étape finale de ce traitement doit conduire à la reconstruction du squelette correspondant au réseau de parois des grains, réseau qui pourra être ensuite implémenté dans un code de calcul.

\section{Modélisation multi-échelles}

La modélisation multi-échelles vise à reproduire la réponse d'une morphologie réelle de mousse comprenant une agglomération de grains, eux-mêmes constitués de cellules microscopiques. À l'échelle mésoscopique, la structure des parois des grains peut être raisonnablement représentée par une approche éléments-finis (FEM); un VER d'un matériau cellulaire est construit à partir d'un réseau de parois denses. Il semble vain de vouloir utiliser le même outil pour modéliser à la fois la structure des parois des grains et la structure plus fine des cellules microscopiques (le nombre d'éléments nécessaires pour représenter ces deux échelles deviendrait phénoménal), une modélisation par eléments discrets (DEM pour discrete element modelling) peut alors être envisagée en parallèle pour représenter la structure cellulaire microscopique. L'étape finale doit être l'intégration du modèle numérique DEM de la structure cellulaire dans le modèle FEM de la structure des grains afin de prendre en compte l'interaction des comportements aux deux échelles. Ce projet numérique nécessite des développements d'outils numériques (intégration DEM/FEM) coûteux en temps et en ressource. Il était nécessaire d'estimer le potentiel de cette méthodologie par des premières investigations numériques. Cet article présente les résultats de simulations numériques en $2 \mathrm{D}$ réalisées aux deux échelles :

- En FEM, la structure des grains a été modélisée par un treillis de parois denses et le comportement des cellules a été homogénéisé, dans un premier temps, par un modèle macroscopique classique. Cette première 
approche, utilisant des modèles simples, vise à reproduire les mécanismes d'endommagement du matériau à l'échelle mésoscopique.

- En DEM, les cellules sont représentées par des particules qui interagissent entre elles par des forces de contact. Une loi de comportement à l'échelle microscopique décrivant la réponse d'une cellule (modèle Gibson) dicte l'évolution de cette force de contact en fonction de l'état de la particule (du comportement élastique à sa densification). Ce modèle DEM a montré l'influence de la microstructure sur la réponse du matériau.

\subsection{Modélisation des grains à l'échelle mésoscopique}

Une première investigation numérique a été menée en 2D en modélisant par éléments-finis la géométrie d'un grain par un polyèdre. Pour résumer les conditions de simulation, un domaine simplifié (hauteur $5,5 \mathrm{~mm}$, largeur $6 \mathrm{~mm}$ sur une profondeur de $1 \mathrm{~mm}$ ) représentatif d'une structure d'une mousse polymère a été représenté (Fig. 13a) avec le code de calcul éléments-finis LS-DYNA. La structure mésoscopique est caractérisée par un ensemble de grains séparés par une structure de parois denses. La porosité des grains constitués de milliers de cellules microscopiques n'est pas représentée par la méthode éléments-finis; pour cette modélisation, la structure des grains est supposée homogène et la densité du grain central est variable $\left(25,90\right.$ et $\left.180 \mathrm{~kg} . \mathrm{m}^{-3}\right)$ par rapport à celle des grains voisins qui reste constante et égale à $70 \mathrm{~kg} . \mathrm{m}^{-3}$. Il est ainsi envisagé d'estimer l'influence du champ de densité sur le champ de déformation.

L'épaisseur $e$ des parois est aussi une variable de la modélisation; trois valeurs ont été choisies $0,02 \mathrm{~mm}$, $0,1 \mathrm{~mm}$ et $0,2 \mathrm{~mm}$ suite aux observations effectuées sur des matériaux cellulaires réels. Le volume de ces parois est donc respectivement de $0,39 \mathrm{~mm}^{3}, 0,78 \mathrm{~mm}^{3}$ et $3,9 \mathrm{~mm}^{3}$. Il correspond donc à $1,1 \%, 2,2 \%$ et $11 \%$ du volume total de l'échantillon poreux. En prenant en compte la masse supplémentaire de ces parois dans un échantillon dont les grains poreux ont tous une densité de $70 \mathrm{~kg} \cdot \mathrm{m}^{-3}$, la densité apparente de l'échantillon comprenant grains et parois est alors égale à 72,74 et $88 \mathrm{~kg} \cdot \mathrm{m}^{-3}$ pour les trois épaisseurs considérées.

Le comportement du matériau des parois est supposé élasto-plastique, le modèle utilisé est la loi 24, *MAT_PIECEWISE_LINEAR_PLASTICITY de LS-DYNA, les paramètres sont le module de Young = $1000 \mathrm{MPa}$, le coefficient de Poisson $=0,1$, la limite élastique $=40 \mathrm{MPa}$ et le module plastique $100 \mathrm{MPa}$. Ces données ont été identifiées par une étude bibliographique $[6]$.

Le comportement de la structure cellulaire microscopique (constituant l'intérieur poreux de chaque grain) est homogénéisé et comprend les trois phases classiques (élastique - plateau en contrainte - densification). Ce comportement peut être représenté par un modèle déjà implémenté dans LS-DYNA; le modèle utilisé est la loi *MAT_LOW_DENSITY_FOAM. Les paramètres de ce

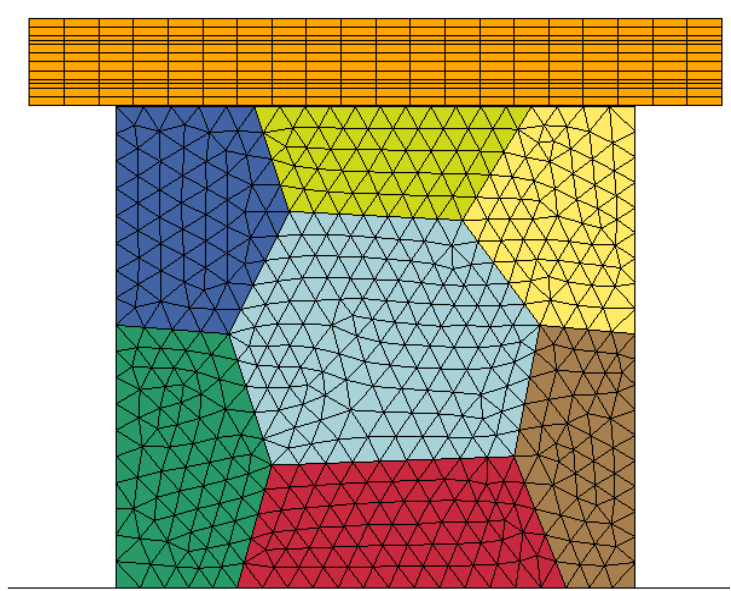

(a)

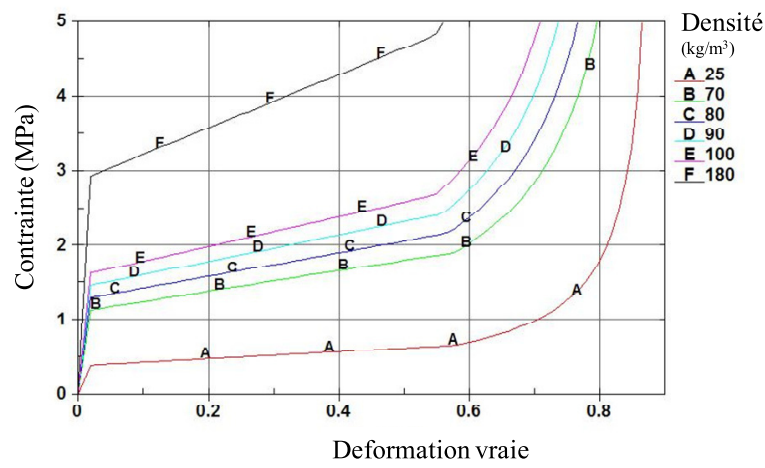

(b)

Fig. 13. (a) Domaine initial modélisé sous LS DYNA. (b) Comportement du matériau cellulaire implémenté dans LSDYNA en fonction de la densité.

modèle dépendent de la densité $\rho$ locale du matériau poreux. La réponse en contrainte-déformation du matériau poreux en fonction de la densité (Fig. 13b) est utilisé pour identifier ce modèle.

Les conditions aux limites sont les suivantes : le domaine numérique est en appui plan avec une surface rigide fixe sur la surface inférieure. Le déplacement de la surface supérieure de l'échantillon est imposé suivant un axe vertical, la vitesse de déplacement de la surface est de $2 \mathrm{~m} . \mathrm{s}^{-1}$. Enfin, les nœuds positionnés sur les quatre surfaces latérales ne peuvent se déplacer que dans les 4 plans considérés. L'objectif final de cette modélisation n'est pas de reproduire une compression uni axiale mais de mettre en évidence les effets de variation d' épaisseur ou de densité locale, les conditions aux limites imposées sur l'échantillon numérique correspondent à une compression en matrice. Ces conditions aux limites particulières ont été choisies pour approcher l'état de sollicitation d'un grain au centre d'une structure poreuse. Ce choix est discutable mais l'objectif de cette modélisation n'est pas de reproduire le comportement d'une mousse sous compression uni axiale mais d'exacerber l'effet de densité locale sur la déformation des grains dans des 

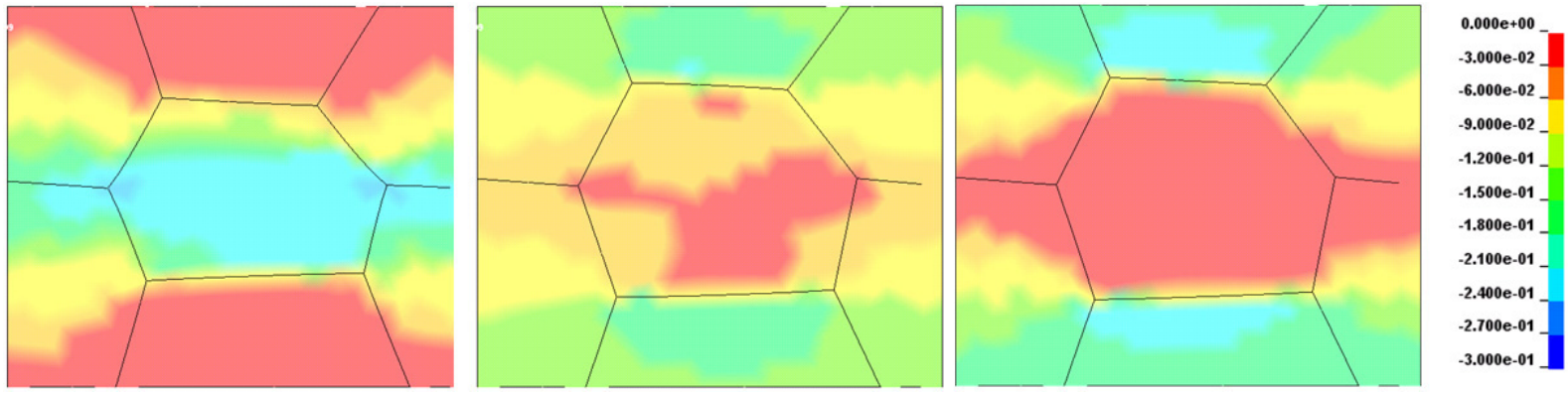

Fig. 14. 3 champs de déformation $\varepsilon_{z}$ ( $z$ étant l'axe de compression) en fonction de la densité $\rho$ du grain central (pour une déformation axiale moyenne de $10 \%$ et une épaisseur de parois nulle) : à gauche) $\rho=25 \mathrm{~kg} \cdot \mathrm{m}^{-3}$, au centre) $\rho=90 \mathrm{~kg} . \mathrm{m}^{-3}$, à droite) $\rho=180 \mathrm{~kg} \cdot \mathrm{m}^{-3}$.
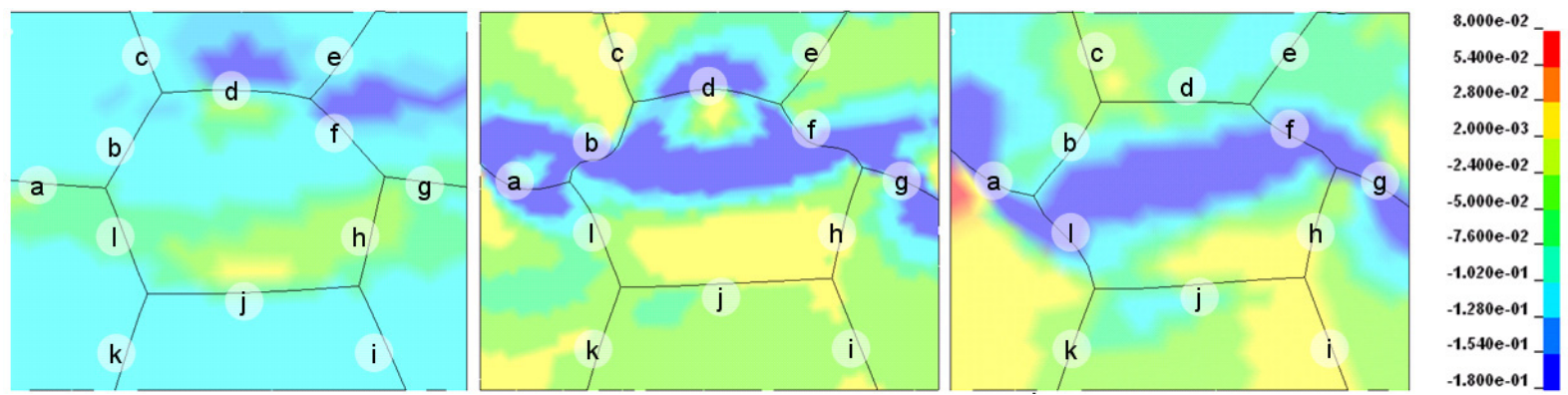

Fig. 15. 3 champs de déformation $\varepsilon_{z}$ ( $z$ étant l'axe de compression) en fonction de l'épaisseur des parois des grains (pour une déformation axiale moyenne de $10 \%$ et un champ de densité homogène), (a) $e=0,02 \mathrm{~mm}$, (b) $e=0,1 \mathrm{~mm},(\mathrm{c}) e=0,2 \mathrm{~mm}$.

conditions de sollicitation « voisines » de celles observées expérimentalement. Un plan d'expériences basé sur des essais numériques a été développé afin de révéler les effets cumulés des deux paramètres densité des grains et épaisseurs de parois sur la localisation des déformations. Les résultats obtenus par ce plan d'expérience peuvent être synthétisés comme suit :

1. L'effet du champ de densité sur l'hétérogénéité du champ de déformation a été démontré numériquement. Comme prévu, la déformation du grain central est d'autant plus importante si sa densité est faible. Sans paroi de grain $(e=0)$, le grain le moins dense se déforme en premier et engendre ensuite la déformation des grains qui sont situés dans le même plan horizontal et perpendiculaire à l'axe de compression (Fig. 14). Ce résultat est évident mais il confirme l'hypothèse d'initiation localisée du dommage et de sa propagation de grain en grain.

2. L'effet de la structure des parois des grains sur la localisation des déformations est confirmé et ce résultat est nouveau (Fig. 15). À densité constante $\rho=70 \mathrm{~kg} \cdot \mathrm{m}^{-3}$, le champ de déformation est particulièrement hétérogène pour les fortes épaisseurs de paroi (Fig. 15c). La modélisation révèle les phénomènes de flambement dans les parois des grains et ce sont ces flambements qui initient cette fois la localisation de déformation. Dans cette configuration, le processus de dégradation du matériau peut être le suivant : pendant la compression, un champ de force s'installe dans le réseau des parois et dans les domaines poreux au cour des grains. Le champ de force augmentant, un premier flambement apparaît sur une des parois et induit une redistribution du champ de force au voisinage de cette paroi. La structure cellulaire des grains s'endommage localement; les valeurs des déformations montrent qu'elle atteint rapidement le plateau en contrainte. Il y a donc au final un transfert de charge vers les autres parois voisines et, en fonction de la géométrie et de l'épaisseur de ces parois, un nouveau flambement apparaît.

3. Ce processus est confirmé par des modélisations couplant les effets de variation de densité et d'épaisseur de paroi (Figs. 16 et 17). Pour des épaisseurs de paroi proches de celles mesurées sur des mousses de polypropylène $(e=0,02 \mathrm{~mm})$, la variation de densité du grain central implique un effet sur le flambement des parois des cellules. Ce ne sont pas les mêmes parois qui flambent en premier : parois b, f, l et h de la figure 17a pour le grain central de faible densité et parois c, e, k et i de la figure $17 \mathrm{c}$ pour le grain central de forte densité. La déformée de ces parois dépend aussi de la densité des grains voisins. Dans cette configuration (faible épaisseur) les modélisations révèlent des flambements multi modaux des parois des grains, phénomènes physiques observés expérimentalement et qui avaient été expliqués par la rigidité latérale de la structure des cellules microscopiques voisines.

Ces modélisations numériques décrivent des processus de dégradation progressifs du matériau cellulaire qui dépendent des effets cumulés du champ de densité des cellules (échelle microscopique) et du réseau des parois des grains (structure mésoscopique). Deux hypothèses 

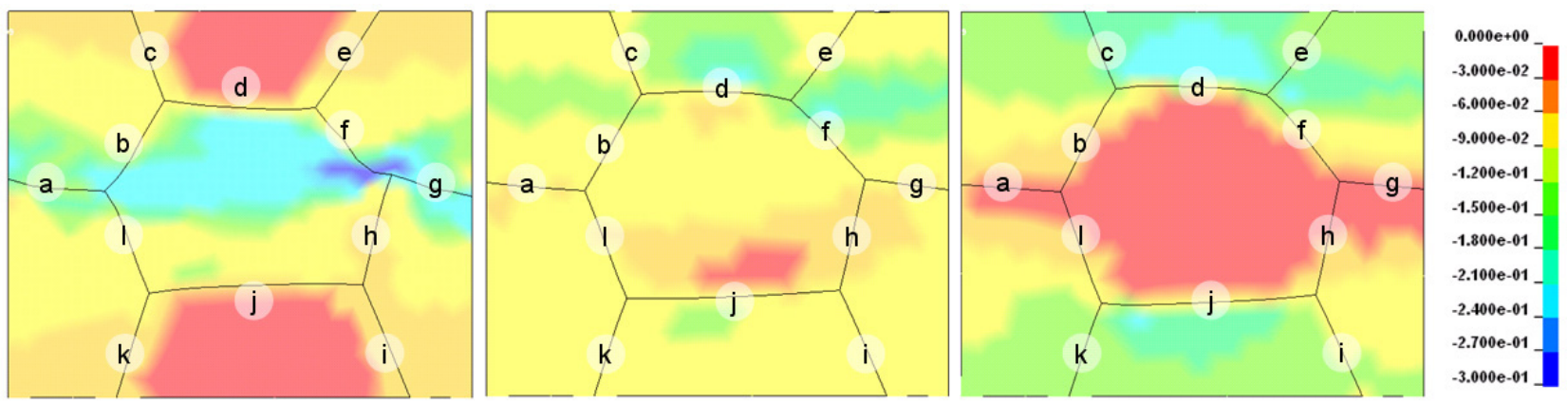

Fig. 16. Champ de déformation $\varepsilon_{z}$ ( $z$ étant l'axe de compression) en fonction de la densité $\rho$ du grain central (avec une épaisseur $e=0,02 \mathrm{~mm}$ ) ; pour une déformation axiale moyenne de $10 \%$, (a) $\rho=25 \mathrm{~kg} \cdot \mathrm{m}^{-3}$, (b) $\rho=90 \mathrm{~kg} \cdot \mathrm{m}^{-3}$, (c) $\rho=180 \mathrm{~kg} \cdot \mathrm{m}^{-3}$.
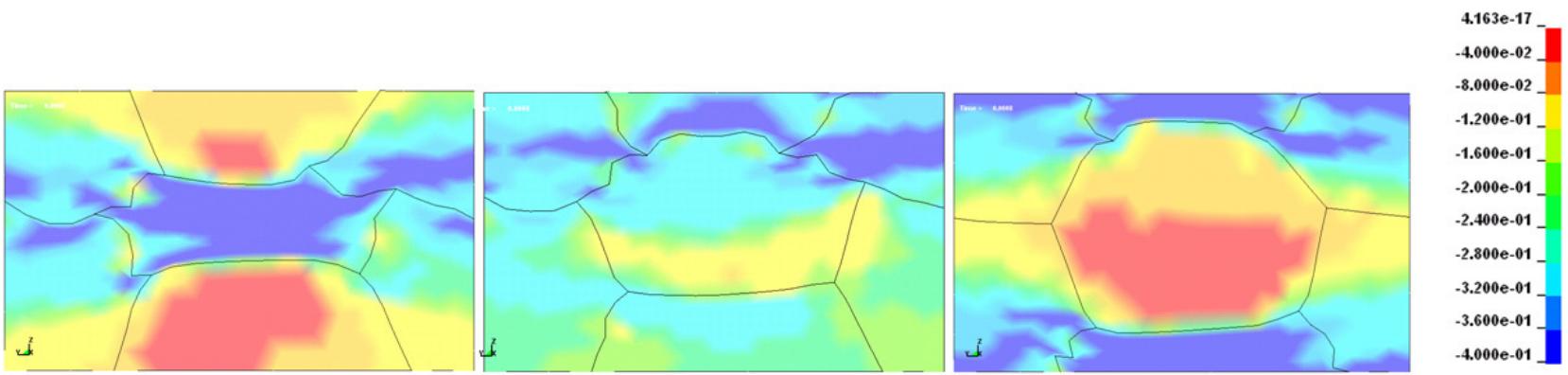

Fig. 17. 3 champ de déformation $\varepsilon_{z}$ ( $z$ étant l'axe de compression) en fonction de la densité $\rho$ du grain central (avec une épaisseur $e=0,02 \mathrm{~mm}$ ) ; Ia) pour une déformation axiale moyenne de $30 \%$, (a) $\rho=25 \mathrm{~kg} \cdot \mathrm{m}^{-3}$, (b) $\rho=90 \mathrm{~kg} \cdot \mathrm{m}^{-3}$, (c) $\rho=180 \mathrm{~kg} \cdot \mathrm{m}^{-3}$.

peuvent être envisagées dans l'initiation du dommage : ou une densité plus faible sur un grain entraîne une localisation du champ de force sur une des parois, ou une paroi plus élancée (donc plus rapidement sujette au flambement) initie une instabilité dans ce champ de forces. Dans tous les cas, le flambement de cette paroi génère ou accentue une localisation de déformation dans les grains voisins, une «plastification» des domaines cellulaires proches ce qui va induire à nouveau le flambement d'une nouvelle paroi...

En terme de contrainte, lorsque le comportement du grain de densité plus faible rentre dans la phase du plateau plastique, la contrainte que peut supporter ce grain devient constante (si on néglige la pente du plateau) ce sont donc les autres grains voisins et leurs parois qui voient leurs contraintes augmenter par simple redistribution. Si la contrainte est telle qu'elle correspond à la contrainte de flambage d'une des parois, alors cette paroi flambe et on assiste à nouveau à une redistribution des contraintes dans la structure qui engendre successivement des flambements de paroi et des effondrements de grain. On peut donc reproduire les bandes de localisation de déformations observées expérimentalement.

Les résultats de ces modélisations multi-échelles du matériau cellulaire montrent donc que la structure des parois des grains et leurs densités intrinsèques ont un effet conjugué sur la déformation du matériau. Ces modélisations ont été cependant calculées en considérant un matériau homogénéisé pour le cœur poreux des grains (échelle des cellules) et le modèle complet doit prendre en compte la morphologie et l'organisation des cellules. Les éléments-finis ne semblent pas adaptés pour représenter simultanément l'échelle plus fine des cellules ainsi que la structure des grains et de leurs parois. Plusieurs auteurs ont déjà modélisé la structure microscopique d'un nombre limité (et raisonnable numériquement parlant) de cellules d'une mousse (mono-échelle) à l'aide d'outils numériques FEM afin de déterminer l'influence de la microstructure sur le comportement macroscopique $[7,12,13]$. Cependant, l'utilisation de ces mêmes outils ne peut être envisagée pour reproduire les parois des milliers de cellules microscopiques constituant un ensemble de grains poreux (échelle mésoscopique) et les parois des grains dans un échantillon (nombre excessif d'éléments). Il est donc raisonnable de proposer d'autres voies numériques pour représenter les deux échelles des matériaux cellulaires envisagés. Une modélisation numérique des cellules microscopiques d'une mousse a donc été développée par la méthode des éléments discrets (DEM). Cette méthode sera ensuite associée à une méthode FEM pour décrire le couplage de comportement entre les deux échelles du matériau.

\subsection{Modélisation à l'échelle microscopique}

La voie numérique DEM est séduisante puisque ces modèles sont constitués par un ensemble de particules (disques, sphères ou bâtonnets) dont l'organisation peut être assez proche d'un agglomérat de cellules microscopiques. La modélisation par éléments discrets semble 

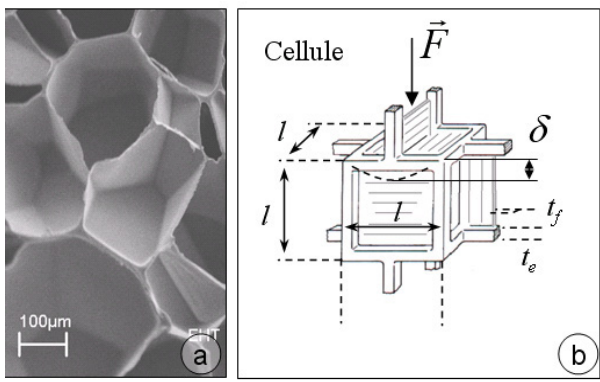

Particule i

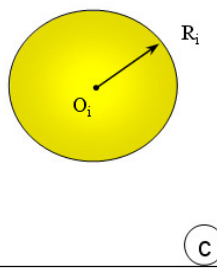

Fig. 18. (a) Cellules réelles ; (b) cellule modélisée par Gibson ; (c) cellule modélisée en DEM.

donc bien adaptée d'un point de vue géométrique mais aussi d'un point de vue sollicitation puisque la méthode est basée sur l'algorithme de dynamique moléculaire qui peut gérer des forces dynamiques entre particules ou cellules $[14,15]$. De plus l'intérêt de cette méthode de modélisation est de prendre en compte la réponse de toutes les particules élémentaires pour mettre en évidence la physique multi-échelles et le comportement macroscopique du domaine, ce qui correspond aux objectifs de la démarche scientifique. Cependant, les modèles DEM couramment utilisés considèrent les particules comme des éléments indéformables. Les forces d'interaction entre deux particules sont modélisées généralement par des forces de contact qui dépendent d'un coefficient de rigidité, d'un coefficient d'amortissement et de la distance relative entre les éléments. Ce modèle de comportement inter particules a donc été modifié pour que les forces interagissant entre les cellules (ou éléments) correspondent au comportement local de la porosité microscopique de la mousse [8].

Selon l'approche envisagée de modélisation DEM, la géométrie des particules reste inchangée et ce sont les forces inter particules qui sont pilotées par une loi de comportement reproduisant la réponse d'une cellule et son interaction avec les cellules voisines. Cette approche nécessite le choix d'un modèle de comportement à l'échelle de la cellule. Plusieurs auteurs ont déjà proposé un modèle de comportement en fonction de la géométrie de la cellule, qui peut être représentée simplement par un cube (modèle de Gibson [6], Fig. 8b) ou par des géométries plus complexes (dodecahedron proposé par Kraynik [12] ou tetrakaidecahedron proposé par William [13]). Dans tous les cas, l'objectif de ces modèles est de transcrire la déformation locale des parois et des arêtes des cellules (par flambage, rotule plastique ou flexion) en un comportement homogénéisé sur la cellule. Notre méthodologie de modélisation mise en ouvre vise à reproduire par une formulation simple des phénomènes physiques complexes; nous avons donc choisi, pour modéliser le comportement d'une cellule en DEM (Fig. 18c), d'utiliser le modèle de Gibson qui décrit simplement la réponse élastique - plateau en contrainte - densification d'une cellule (Fig. 19a) en fonction de sa géométrie. Ce modèle a été adapté pour que ses caractéristiques dépendent de la densité de la particule.

\subsubsection{Le modèle micromécanique}

Dans le cas de modélisation classique par la méthode des éléments discrets, un matériau constitué d'un assemblage de grains indéformables est localement soumis à un champ de contrainte hétérogène même si la sollicitation macroscopique appliquée sur le matériau est une pression constante. Cet état local n'est pas dû à une hétérogénéité du comportement du matériau des grains mais à la géométrie de la structure du matériau granulaire. Cette propriété a été exploitée pour introduire des localisations de comportement dans les matériaux cellulaires sollicités en compression. Les cellules sont en interaction par des forces locales et la localisation des déformations et des contraintes est introduite par la variabilité locale de la taille des cellules. La variation de la dimension des cellules introduit une distribution des contacts entre les cellules et donc des forces qui peuvent se transmettre entre les porosités. De plus, nous supposons que la réponse mécanique d'une cellule dépend de sa dimension. Pour chaque cellule, son comportement en compression - sa force de réaction - est établi selon le modèle proposé par Gibson et Ashby à partir des paramètres qui sont une raideur élastique $K_{\mathrm{e}}$, une raideur plateau $K_{\mathrm{p}}$, une force seuil $f_{\mathrm{pl}}$ (initiation du plateau), et une raideur de densification $K_{\mathrm{d}}$ qui débute pour une limite en déformation $\varepsilon_{\mathrm{d}}$ (Fig. 19a). Chaque cellule est donc représentée par un disque et l'interaction entre deux disques est calculée à partir de la réponse mécanique des cellules (dont les paramètres dépendent de la dimension des cellules). Pour chaque phase de comportement, la réponse macroscopique en force $F$ fonction du déplacement $\delta$ est donc déterminée par les déformations supposées de la cellule élémentaire selon Gibson et Ashby. Il y a donc une relation directe entre les forces entre les éléments (disques) du domaine granulaire et le comportement des cellules modélisées.

Pour modéliser la réponse mécanique de la cellule élémentaire, Gibson et Ashby introduisent un paramètre $\phi$ qui traduit la fraction de matériau contenue dans les arêtes des cellules tandis que la fraction restante $(1-\phi)$ correspond à la fraction de matière des parois. L'analyse géométrique des cellules permet l'identification de ce coefficient. Une campagne d'observation sur images MEB de la structure microscopique des mousses de polypropylène étudiées fournit une valeur de $\phi$ voisine de 0,1 .

Dans le domaine élastique, Gibson et Ashby définissent la raideur élastique homogénéisée de la cellule à partir de la flexion des arêtes de la cellule en fonction de la force $F$ imposée :

$$
K_{\mathrm{e}}=\left(K_{1} \phi^{2}\left(\frac{\rho}{\rho^{*}}\right)^{2}+K_{2}(1-\phi)\left(\frac{\rho}{\rho^{*}}\right)\right) E^{*} l
$$

avec $\rho$ la densité de la cellule (de taille $l$ ), $\rho^{*}$ et $E^{*}$ la densité et le module de Young du matériau dense. Les coefficients $K_{1}$ et $K_{2}$ sont fournis par Gibson et Ashby sur un certain nombre de mousses, nous avons préféré identifier 


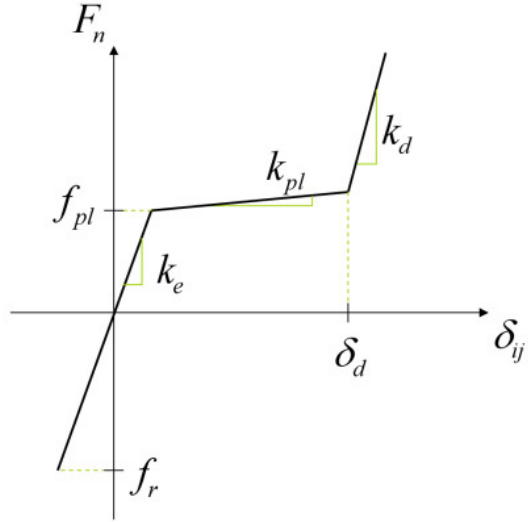

(a)

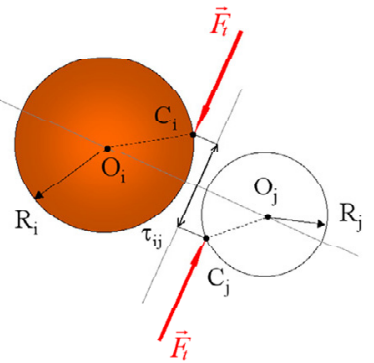

(b)

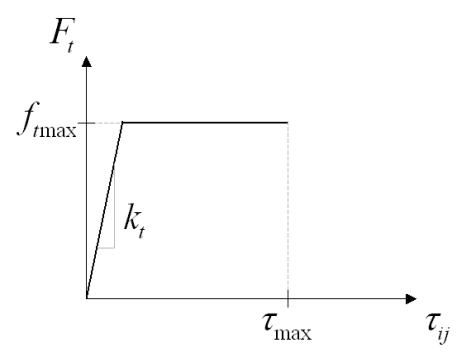

b)

Fig. 19. (a) Modèle micro mécanique proposé par Gibson pour la prise en compte des sollicitations de traction-compression. (b) Modèle micromécanique pour la prise en compte des sollicitations de cisaillement.

ces coefficients à partir de nos résultats expérimentaux. L'effet de géométrie de la cellule sur cette raideur dépend donc de la variable $\phi$, de la densité $\rho$ de la cellule et de la longueur caractéristique $l$. Pour ces modélisations, $\phi$ est imposé constant (la proportion d'arêtes et de parois dans une cellule est constante) et la longueur caractéristique $l$ est considérée égale au diamètre moyen $D$ des cellules du domaine. Par hypothèse, la raideur élastique de la particule dépend donc de la variable densité de la cellule.

Les autres paramètres du modèle de Gibson et Ashby sont identifiés de manière similaire. Nous proposons par la suite les relations d'identification de ces paramètres en prenant en compte la dimension de la particule $(l=2 \mathrm{R})$ et en considérant la densité de la cellule comme la variable d'entrée.

Dans la phase plateau, les mécanismes principaux sont le flambement des parois et l'apparition de rotule plastique. À partir de ces mécanismes, Gibson et Ashby proposent un modèle qui définit la limite élastique en effort en fonction de la taille de la cellule :

$$
f_{\mathrm{pl}}=\left(f_{1} \phi^{3 / 2}\left(\frac{\rho}{\rho^{*}}\right)^{3 / 2}+f_{2}(1-\phi)\left(\frac{\rho}{\rho^{*}}\right)\right) \sigma_{\mathrm{pl}}^{*}(2 R)^{2}
$$

Avec $\sigma_{\mathrm{pl}}^{*}$ la contrainte de limite élastique. Cette contrainte ainsi que les paramètres $f_{1}$ et $f_{2}$ sont identifiés expérimentalement sur les matériaux de l'étude.

Les mécanismes de déformation pendant le plateau en contrainte sont relativement complexes, ils comprennent une progression du flambement (en grand déplacement) des arêtes et des parois, des tensions et compressions sur certaines faces de la cellule et des compressions de gaz. Nous avons proposé une relation homogénéisée à l'échelle de la cellule qui permet d'établir une raideur équivalente de la cellule pendant la phase plateau en fonction du module tangent macroscopique $E_{\mathrm{pl}}^{*}$ de la mousse :

$$
K_{\mathrm{pl}}=\left(K_{\mathrm{pl} 1} \phi^{4}\left(\frac{\rho}{\rho^{*}}\right)^{4}+K_{\mathrm{pl} 2}(1-\phi)\left(\frac{\rho}{\rho^{*}}\right)\right) E_{\mathrm{pl}}^{*} 2 R
$$

Enfin la densification de la cellule s'initie pour un déplacement $\delta_{\mathrm{d}}$ limite qui est établi grâce à Gibson et Ashby par la relation empirique :

$$
\delta_{\mathrm{d}}=\left(1-1,4\left(\frac{\rho}{\rho^{*}}\right)\right) 2 R
$$

Pour cette phase ultime le comportement de la cellule effondrée est considéré équivalent à celui du matériau dense, la raideur de densification est supposée identique à celle du matériau constitutif. Cette hypothèse est simpliste évidemment; cela revient à considérer l'empilement des parois de cellules écrasées comme un matériau homogène.

Le modèle de Gibson et Ashby est forcément critiquable; les hypothèses sur les déformations sont simples, certaines relations sont empiriques... Des modèles plus sophistiqués permettent certainement de décrire avec plus de justesse les effets des déformations des parois et arêtes d'une cellule sur sa réponse contrainte-déformation (ou force-déplacement). Cependant, par cette approche numérique qui vise à établir le comportement de la structure microscopique des cellules, il n'est pas nécessaire de descendre à une échelle encore plus fine afin d'analyser la réponse de la géométrie d'une cellule, nous nous contentons d'établir le comportement homogénéisé d'une cellule (à l'échelle microscopique) en fonction de sa taille.

Chaque cellule a donc un comportement propre en compression. L'interaction entre deux particules $i$ et $j$ met en jeu les paramètres identifiés précédemment en fonction de la densité de chaque particule. La force d'interaction est calculée à partir d'une raideur équivalente aux raideurs de ces deux particules.

La réponse de la cellule à une sollicitation de traction est supposée élastique fragile (de valeur seuil $f_{\mathrm{r}}$ ). Par hypothèse, la rigidité en tension est supposée égale à la rigidité en compression (Fig. 19a).

La modélisation doit aussi prendre en compte le comportement du matériau pour les sollicitations de cisaillement. Nous proposons de décrire le comportement en cisaillement entre deux cellules par un comportment elasto plastique parfait (Fig. 19b). La partie élastique est définie 
par une rigidité tangentielle $K_{\mathrm{t}}$ : quand une force de cisaillement apparait entre deux cellules, les points en contact $C_{i}$ et $C_{j}$ (initialement en coïncidence) glissent et le déplacement relatif entre ces deux points induit un effort tangentiel de rappel $F_{\mathrm{t}}$. Cet effort tangentiel est limité à un effort $f_{\max }$. Passé ce seuil, le comportement est supposé plastique parfait jusqu'à la rupture du contact entre les deux cellules.

\subsubsection{Quelques résultats significatifs}

Pour une première approche, l'influence de la densité et de la morphologie - à l'échelle microscopique de la mousse sur son comportement a été étudiée par des simulations 2D. Il est évident que des effets tridimensionnels peuvent être mis en évidence sur le comportement du matériau - d'un point de quantitatif - mais ces effets peuvent être négligés si l'étude numérique 2D se limite à des conclusions qualitatives. Dans l'étude de l'influence de la microstructure sur le comportement macroscopique d'une mousse de polypropylène (par analyse de mesures microtomographiques), il a été montré que de premières conclusions pouvaient être proposées via une analyse des résultats en 2D [10], et que ces conclusions pouvaient être confirmées par le traitement complet en $3 \mathrm{D}$ des résultats [16]. Pour cette première approche de modélisation DEM de matériaux cellulaires, les simulations ont donc été effectuées en 2D afin de réduire les temps de calcul. L'échantillon modélisé est un domaine bidimensionnel dont les caractéristiques sont les suivantes :

- Un déplacement vertical est imposé sur la face supérieure; La vitesse de déplacement de la face supérieure est de $1,6 \times 10^{-4} \mathrm{~m} . \mathrm{s}^{-1}$.

- La face inférieure est fixe.

- Les murs - ou mors de compression - supérieurs et inférieurs sont composés de particules rigides. Cette modélisation des mors de compression permet d'utiliser la même méthode numérique de recherche des contacts au niveau des conditions aux limites (contact mors de compression/mousse) que celle utilisée pour rechercher les contacts entre les éléments du matériau cellulaire modélisé. La taille des éléments des murs peut avoir une influence sur le coefficient de frottement entre mors de compression et le matériau cellulaire. Cet effet ne sera pas étudié dans cet article.

- Des conditions aux limites périodiques sont imposées sur les faces latérales (gauche et droite) du domaine. Chaque élément - ou particule - d'une de ces faces peut donc virtuellement être en contact avec une ou plusieurs particules de l'autre face latérale. Cela implique que virtuellement, le domaine est étendu à l'infini; cette condition aux limites conduit en fait à réaliser une compression confinée (en matrice) sur une zone réduite de ce domaine infini. Le nombre de particules est ainsi limité et par conséquent le temps de calcul est réduit. Ces choix de conditions aux limites particulières influent sur la réponse macroscopique du matériau modélisé mais l'objectif de ces premières

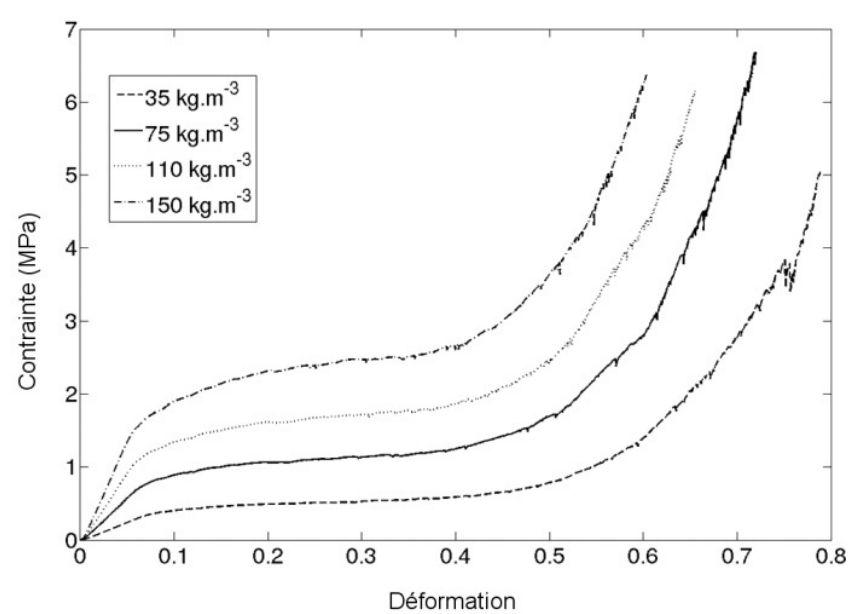

Fig. 20. Réponse macroscopique contrainte-déformation d'un domaine DEM en fonction de la densité des particules.

modélisations était de mettre en évidence l'effet de la morphologie de la mousse sur son comportement et non pas de retrouver la réponse d'un matériau cellulaire soumis à une sollicitation mécanique particulière.

Les modèles éléments discrets sont souvent utilisés pour modéliser et expliquer des phénomènes physiques, moins pour quantifier la réponse d'un matériau ou d'une structure. Cependant, les modélisations DEM effectuées fournissent une réponse macroscopique contrainte déformation du matériau cellulaire en fonction de la densité proche de celle mesurée expérimentalement. La réponse modélisée (Fig. 20) élastique - plateau - densification de la mousse polymère montre des seuils et des raideurs qui dépendent de la densité et dont l'évolution est cohérente avec les mesures expérimentales (Fig. 2a). Les résultats expérimentaux ont été présentés dans la première partie de cet article [1], ils montrent clairement la sensibilité du comportement macroscopique du matériau en fonction de la densité. Seule la longueur du plateau - en termes de déformation - est plus faible que celle observée expérimentalement. Des modélisations en 3D devraient permettre d'améliorer la corrélation entre les résultats numériques et expérimentaux.

À l'échelle locale, l'effet de taille et d'organisation des cellules dans le matériau cellulaire a aussi été révélé en définissant un domaine numérique constitué de particules dont les distributions des diamètres (et donc leurs réponses) suivent une loi gaussienne coupée avec un écart type de 0,2. La densité de chaque particule est constante (quelle que soit sa taille) et c'est donc bien uniquement l'effet de distribution de cette taille dans le domaine qui est évalué. Ces particules de tailles variables ont été réparties de manière aléatoire (Fig. 21) ou en imposant un gradient (Fig. 22).

\section{Remarques:}

Les cartographies des simulations DEM présentent l'évolution du domaine de particules en fonction de la déformation moyenne imposée. Un code couleur a été 

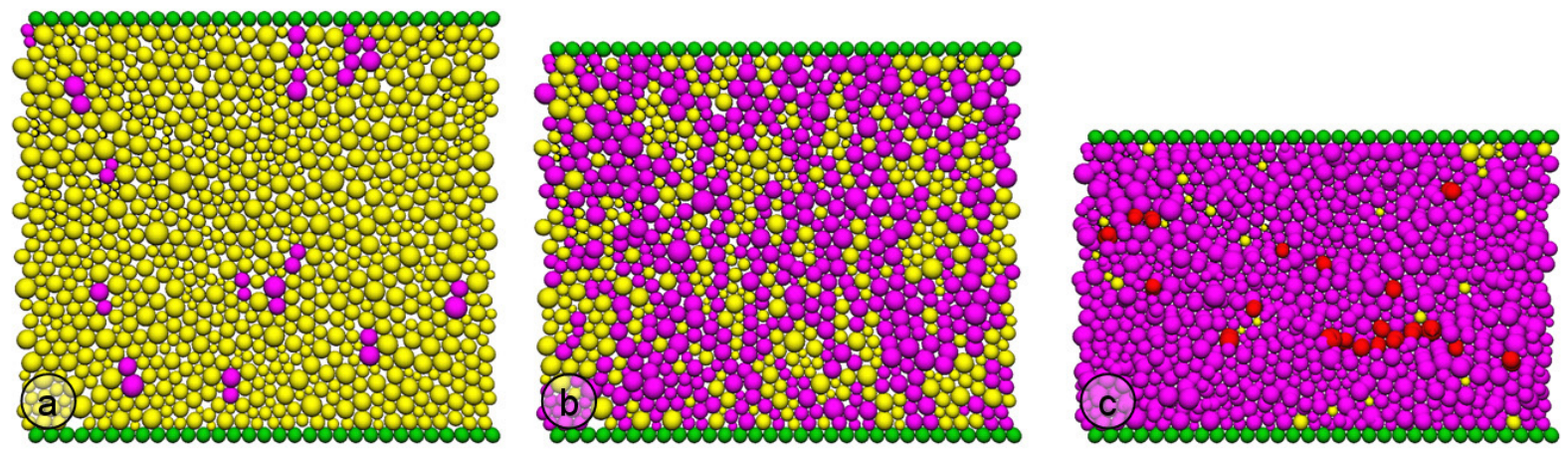

Fig. 21. Évolution du domaine de particules pour une distribution spatiale aléatoire des cellules de taille variable (pour trois valeurs de déformation : (a) $\varepsilon=0,05$; (b) $\varepsilon=0,12$, (c) $\varepsilon=0,32$ ).
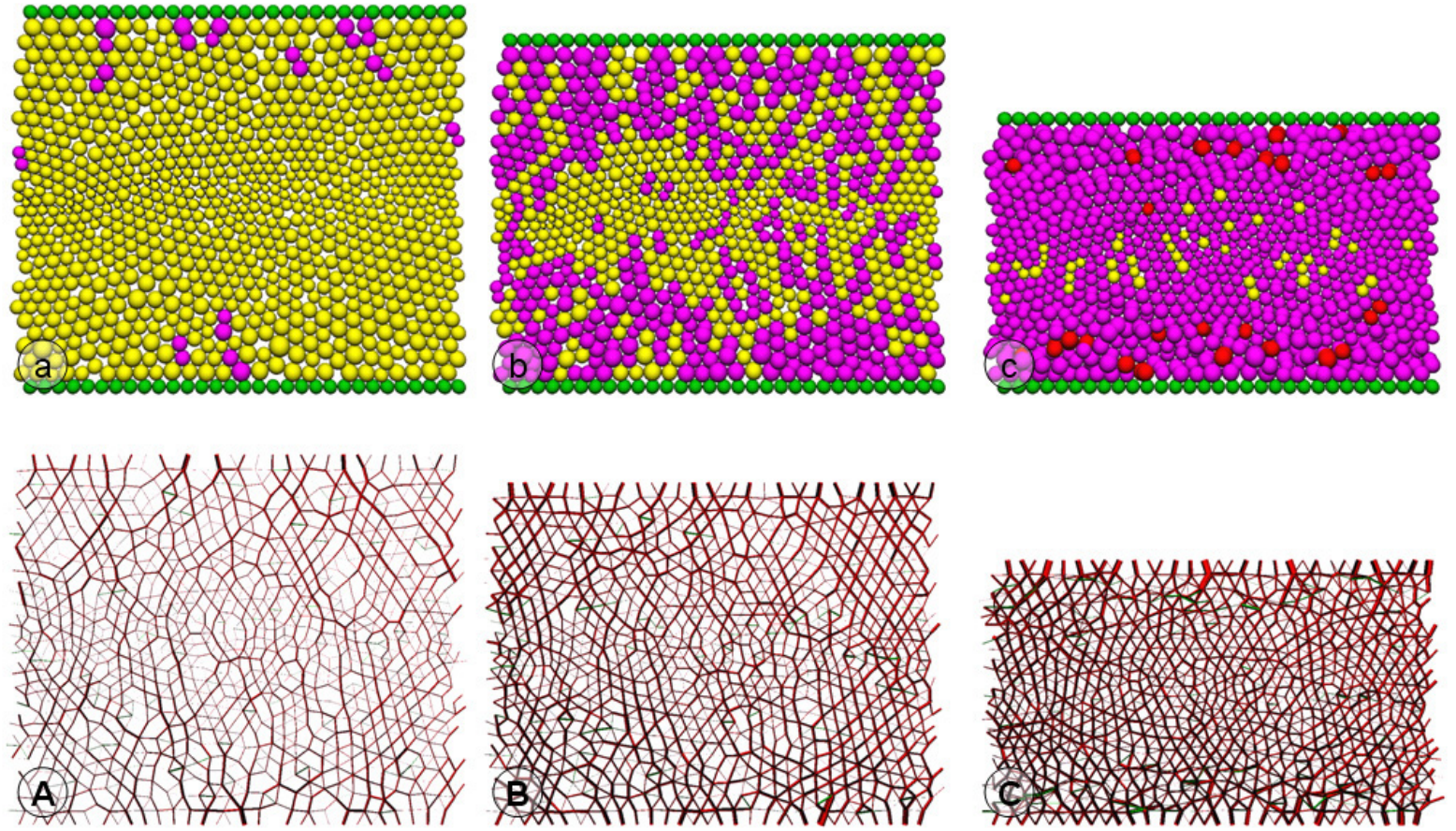

Fig. 22. Évolution du domaine de particules et du champ de forces pour une distribution spatiale à gradient des cellules de taille variable (pour trois valeurs de déformation : (a) $\varepsilon=0,05$; (b) $\varepsilon=0,12$; (c) $\varepsilon=0,32$ ).

établi afin d'estimer l'état de la particule : le jaune correspond à l'état élastique, le violet au plateau en contrainte de couleur et le rouge à la phase de densification. Il est ainsi possible d'évaluer le déplacement de ces particules et leurs états. Dans les cartographies de champ de forces un segment représente la force entre deux particules, l'épaisseur de ce segment indique l'intensité de la force.

La distribution aléatoire des particules de taille variable montre une déformation et un dommage diffus de ces cellules (Fig. 21b). Il n'existe pas de localisation particulière. La microstructure générée dans ces conditions est proche de celle visualisée sur une mousse mono échelle de polyuréthane. Aucune localisation n'a été constatée sur ce type de matériau (Figs. 21b et c).

L'organisation particulière des cellules en fonction de leur diamètre (Fig. 22a) est proche des gradients de taille qui peuvent être observés sur des mousses de polypropylène industrielles (fabriquées par JSP ou Knaupf). Dans ce cas, le dommage apparaît de manière localisée dans les zones où les particules sont les plus grosses (Figs. 22b et c), malgré le fait qu'elles aient toutes les mêmes caractéristiques mécaniques (elles sont toutes de même densité). En fait, l'organisation particulière de ce domaine numérique montre que le nombre de contacts entre particules est d'autant plus faible que les cellules sont de taille importante. Dans les zones où les cellules sont les plus grosses, la force appliquée sur l'échantillon doit être transmise par un nombre plus faible de cellules, les forces entre cellules sont donc plus élevées (Fig. 22A) et atteignent plus rapidement la force seuil de plateau. Le champ de forces présenté (Figs. 22B et C) à différents instants de la déformation explique en fait ce phénomène : les forces sont plus importantes dans les zones de plus grosses particules 

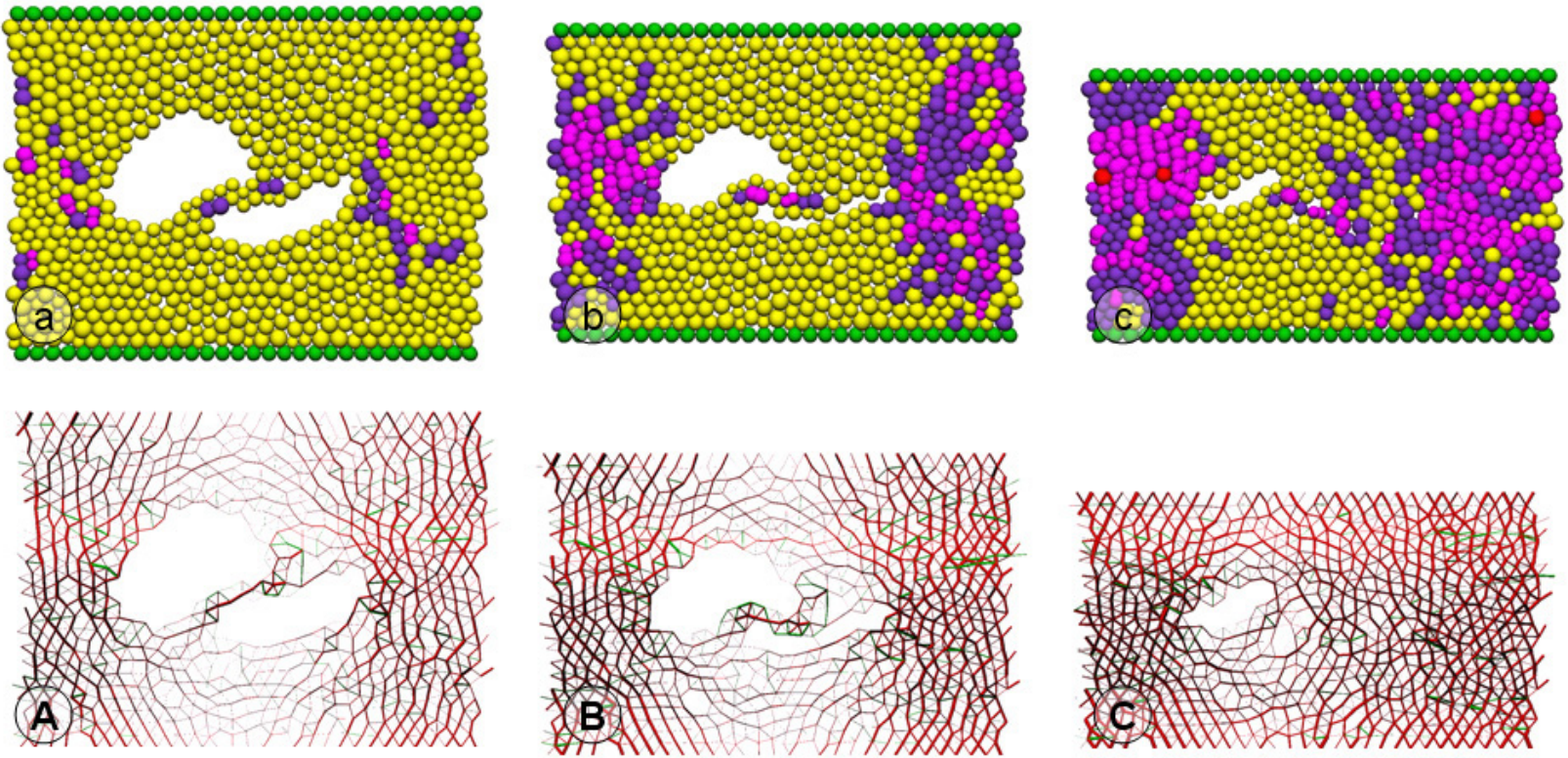

Fig. 23. Évolution du domaine de particules (comprenant un défaut macroscopique) et du champ de forces pour une distribution spatiale à gradient des cellules de taille variable (pour trois valeurs de déformation : (a) $\varepsilon=0,05 ;(\mathrm{b}) \varepsilon=0,12 ;(\mathrm{c}) \varepsilon=0,32$ ) .

puisque les contacts interparticulaires sont moins nombreux. Les seuils en efforts étant les mêmes pour cette modélisation, les dommages sont atteints plus rapidement dans ces zones localisées.

La simulation par élements discrets de la structure microscopique permet d'estimer la réponse de nombreuses microstructures qui se différencient selon la taille variable des cellules, leurs caractéristiques mécaniques, la distribution de ces paramètres, l'organisation des cellules dans le domaine, etc. Les variables d'entrée (causes) sont multiples et certaines configurations ont montré les conséquences aux échelles microscopique (effet de localisation) et macroscopique (réponse contrainte déformation). Ces résultats intéressent bien évidemment les fabricants de matériaux cellulaires qui visent à améliorer les performances de leurs produits.

Par extension, la modélisation DEM de la structure microscopique d'une mousse a permis d'étudier l'influence des défauts (sous forme de larges bulles) dans leur architecture. L'effet de ces bulles macroscopiques sur le comportement du matériau est difficile à atteindre expérimentalement. Grâce à cette approche numérique, les simulations d'un domaine de particules incluant des défauts macroscopiques montrent une forte localisation des dommages (Fig. 23). Le champ de force doit « contourner $»$ le défaut et cette redistribution locale des efforts entraîne des sollicitations plus importantes sur les cellules voisines (situées précisément dans le plan perpendiculaire à l'axe de compression). Le dommage s'initie donc en premier dans cette zone. Pendant la compression, il se propage parallèlement à l'axe de sollicitation (Fig. 23b), un couloir de particules est protégé par l'élasticité du défaut (Fig. 23c). Le champ de force s'homogénéise pendant la fermeture ou écrasement de la bulle macroscopique (Fig. 23C).

\section{Conclusion}

Ce deuxième volet dans la méthodologie de modélisation multi-échelles de matériaux cellulaires sous sollicitations dynamiques était une étape d'identification de phénomènes et de modélisation numérique. Les observations faites aux différentes échelles montrent l'apparition de localisation de déformation pendant la compression; elles apparaissent à la surface du matériau, sont visibles après impact sur des photographies de la structure aux différentes échelles. Cette dégradation localisée s'accompagne (ou s'initie) par le flambement des parois des cellules (à l'échelle mésoscopique) et celles des grains (à l'échelle microscopique). Le même mécanisme est donc à l'origine de la dégradation des deux échelles du matériau et apparaît de manière couplée.

La modélisation multi-échelles proposée a pour objectif de reproduire numériquement la réponse du matériau cellulaire en fonction de sa morphologie. Cette modélisation est mixte, la structure mésoscopique des grains est représentée en éléments-finis et celle des cellules, à l'échelle microscopique, en éléments discrets. Les simulations (utilisant ces deux voies numériques) ont été effectuées séparément aux deux échelles et montrent la faisabilité de la démarche, un verrou scientifique est encore le couplage entre les deux modélisations (intégration du modèle DEM dans le réseau mésoscopique FEM). Les simulations numériques FE d'un volume élémentaire de mousse comprenant un nombre limité de grains utilisaient un matériau homogène continu pour représenter la structure des cellules. Cette étape a confirmé les hypothèses déduites des observations expérimentales : le champ de déformation, les localisations de dommage dépendent de l'effet cumulé de la structure mésoscopique des parois de grains et de leur densité. Grâce à ces 
modélisations, des processus de propagation de dommage ont pu être expliqués. Cependant, puisque la structure des cellules est considérée dans ces simulations comme un matériau homogène, la variabilité de taille et de comportement des cellules ne peut être prise en compte. Les localisations de comportement à l'échelle microscopique ne sont pas considérées et c'est bien sûr l'enjeu majeur de l'intégration du modèle éléments discrets dans cette représentation $\mathrm{FE}$.

En conclusion sur l'approche éléments discrets, les simulations numériques montrent que cette voie de modélisation est adaptée aux matériaux cellulaires et donnent des résultats encourageants tant à l'échelle de la cellule que macroscopiquement parlant. Les localisations qui apparaissent en fonction de la dispersion de taille des cellules et de leurs organisations dans l'architecture du matériau sont reproduites numériquement. La réponse macroscopique de l'échantillon numérique est globalement conforme aux mesures expérimentales. Le couplage des deux modèles FEM et DEM sera finalement nécessaire afin de reproduire in fine la réponse globale de la mousse multi-échelles à une sollicitation dynamique. Ce modèle complet à l'échelle macroscopique pourra être utilisé dans les codes de calcul afin de prédire la réponse d'une structure complète à une sollicitation dynamique (crash, impact, choc...).

Finalement, cette approche de modélisation multiéchelles d'une mousse pourra être utilisée pour évaluer l'influence des nombreux paramètres de microstructure intervenant sur la réponse du matériau aux différentes échelles. Cette étude sera poursuivie en associant partenaires universitaires et industriels dans le développement de procédés de fabrication de matériaux cellulaires, l'objectif final étant d'évaluer l'influence des procédés de fabrication et de mise en forme sur la microstructure de ces matériaux et donc sur leurs performances attendues.

\section{Références}

[1] P. Viot, Comportement des matériaux cellulaires sous sollicitations dynamiques. Partie 1 : approche macroscopique, Mécanique \& Industries 11 (2011) 1-23

[2] P. Chambon, E. Cloutet, H. Cramail, Synthesis of coreshell polyurethane - poly(dimethylsiloxane) particles in supercritical carbon dioxide, Macromolecules 37 (2004) $5856-5859$

[3] C. Mabille, V. Schmitt, Ph. Gorria, F. Leal Calderon, V. Faye, B. Déminière, J. Bibette, Rheological and shearing conditions for the preparations of monodisperse emulsions, Langmuir 16 (2000) 422-429
[4] F. Leal Calderon, T. Stora, O. Mondain-Monval, P. Poulin, J. Bibette, Direct measurement of colloidal forces, Phys. Rev. Lett. 72 (1994) 2959

[5] A. García Loera, F. Cara, M. Dumon, JP Pascault, Porous epoxy thermosets obtained by a polymerizationinduced phase separation process of a degradable thermoplastic polymer, Macromolecules 35 (2002) 6291-6297

[6] L. Gibson, F. Ashby, Cellular solids. Structures and properties, édition : Cambridge Solid State Science Series, 1997

[7] N.J. Mills, Introduction to polymer foam microstructure Polymer Foams Handbook, 2007, pp. 1-18

[8] P. Viot, R. Bouix, I. Iordanoff, J.L. Lataillade, Deformation localisation modelling of polymer foam microstructure under compression: a new approach by discrete element modelling, Compos. Struct. 92 (2010) 585592

[9] J.A. Reglero, Ruiz P. Viot, M. Dumon, Foaming behaviour and compressives properties of microcellular nanostructured polystyrene, Cellular Polymers 28 (2009) 363385

[10] P. Viot, D. Bernard, E. Plougonven, Polymeric foam deformation under dynamic loading by the use of the microtomographic technique, J. Mater. Sci. 42 (2007) 72027213

[11] E. Plougonven, D. Bernard, P. Viot, Quantitative analysis of the deformation of polypropylene foam under dynamic crash loading. Progress in Biomedical Optics and Imaging - Proc. SPIE 6318 (2006)

[12] A.D. Kraynik, W.E. Warren, The elastic behaviour of low density cellular plastics chapter 7 I low density cellular plastics, N.C. Hilyard, A. Cunningham (eds), Chapmann and Hall, London, 1994

[13] R.E. Williams, Space-filling polyhedron: its relation to aggregates of soap bubbles, plant cells, and metal crystallites, Science $161276-277$

[14] N. Fillot, I. Iordanoff, Y. Berthier, A granular dynamic model for the degradation of material, ASME J. Trib. 126 (2004) 606-614

[15] I. Iordanoff, B. Seve, Y. Berthier, Solid third body analysis using a discrete approach: influence of adhesion and particle size on the macroscopic behavior of the contact, ASME J. Trib. 124 (2002) 530-538; (2002 ASME J. Trib. Best Paper Award).

[16] P. Viot, E. Plougonven, D. Bernard, Microtomography on polypropylene foam under dynamic loading. $3 \mathrm{~d}$ analysis of bead morphology evolution. Composites part A 39 (2008) $1266-1281$

[17] A. Desforges, H. Deleuze, O. Mondain-Monval, R. Backov, Palladium nanoparticle generation within microcellular polymeric foam and size dependence under synthetic conditions, Ind. Eng. Chem. Res. 44 (2005) 8521-29 